\title{
A Subarcsecond Near-infrared View of Massive Galaxies at $z>1$ with Gemini Multi-conjugate Adaptive Optics
}

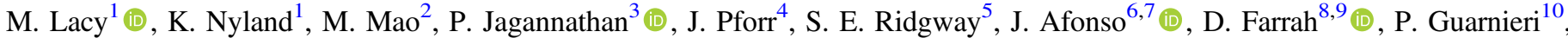 \\ E. Gonzales-Solares $^{11}$, M. J. Jarvis ${ }^{12,13}$, C. Maraston ${ }^{10}$, D. M. Nielsen ${ }^{14}$, A. O. Petric ${ }^{15}$ (D), A. Sajina ${ }^{16}$ (D), J. A. Surace ${ }^{17}$ (D), and \\ M. Vaccari ${ }^{13,18}$ (iD \\ ${ }^{1}$ National Radio Astronomy Observatory, 520 Edgemont Road, Charlottesville, VA 22903, USA \\ 2 Jodrell Bank Centre for Astrophysics, Alan Turing Building, School of Physics and Astronomy, The University of Manchester, \\ Oxford Road, Manchester, M13 9PL, UK \\ ${ }^{3}$ National Radio Astronomy Observatory, 1003, Lopezville Road, Socorro, NM 87801, USA \\ ${ }^{4}$ ESA/ESTEC Keplerlaan1, 2201 AZ, Noordwijk, The Netherlands \\ ${ }^{5}$ National Optical Astronomy Observatory, 950 North Cherry Avenue, Tucson, AZ, 85719, USA \\ ${ }^{6}$ Instituto de Astrofísica e Ciências do Espaço, Universidade de Lisboa, OAL, Tapada da Ajuda, PT1349-018 Lisboa, Portugal \\ ${ }^{7}$ Departamento de Física, Faculdade de Ciências, Universidade de Lisboa, Edifício C8, Campo Grande, PT1749-016 Lisbon, Portugal \\ ${ }^{8}$ Department of Physics and Astronomy, University of Hawaii, 2505 Correa Road, Honolulu, HI 96822, USA \\ ${ }^{9}$ Institute for Astronomy, 2680 Woodlawn Drive, University of Hawaii, Honolulu, HI 96822, USA \\ ${ }^{10}$ Institute of Cosmology and Gravitation, Dennis Sciama Building, Burnaby Road, Portsmouth PO1 3FX, UK \\ ${ }_{11}$ Institute of Astronomy, Madingley Road, Cambridge CB3 OHA, UK \\ ${ }^{12}$ Astrophysics, Department of Physics, Keble Road, Oxford OX1 3RH, UK \\ ${ }^{13}$ Department of Physics and Astronomy, University of the Western Cape, Private Bag X17, 7535, Bellville, Cape Town, South Africa \\ ${ }^{14}$ Dept. of Astronomy, University of Wisconsin-Madison, 475 N. Charter Street, Madison, WI 53706, USA \\ ${ }^{15}$ CFHT Corporation, 65-1238 Mamalahoa Highway, Kamuela, HI 96743, USA \\ ${ }^{16}$ Department of Physics and Astronomy, Tufts University, 212 College Avenue, Medford, MA 02155, USA \\ ${ }^{17}$ Spitzer Science Center, California Institute of Technology, Pasadena, CA 91125, USA \\ 18 INAF-Istituto di Radioastronomia, via Gobetti 101, I-40129 Bologna, Italy \\ Received 2017 September 22; revised 2018 June 22; accepted 2018 July 3; published 2018 August 24
}

\begin{abstract}
We present images taken using the Gemini South Adaptive Optics Imager (GSAOI) with the Gemini Multiconjugate Adaptive Optics System (GeMS) in three $2 \operatorname{arcmin}^{2}$ fields in the Spitzer Extragalactic Representative Volume Survey. These GeMS/GSAOI observations are among the first $\approx 0$ " 1 resolution data in the near-infrared spanning extragalactic fields exceeding 1.5 in size. We use these data to estimate galaxy sizes, obtaining results similar to those from studies with the Hubble Space Telescope, though we find a higher fraction of compact starforming galaxies at $z>2$. To disentangle the star-forming galaxies from active galactic nuclei (AGNs), we use multiwavelength data from surveys in the optical and infrared, including far-infrared data from Herschel, as well as new radio continuum data from the Australia Telescope Compact Array and Very Large Array. We identify ultraluminous infrared galaxies (ULIRGs) at $z \sim 1-3$, which consist of a combination of pure starburst galaxies and AGN/starburst composites. The ULIRGs show signs of recent merger activity, such as highly disturbed morphologies and include a rare candidate triple-AGN. We find that AGNs tend to reside in hosts with smaller scale sizes than purely star-forming galaxies of similar infrared luminosity. Our observations demonstrate the potential for MCAO to complement the deeper galaxy surveys to be made with the James Webb Space Telescope.
\end{abstract}

Key words: galaxies: evolution

Supporting material: machine-readable table

\section{Introduction}

Our current understanding of the evolution of massive galaxies invokes their formation at high redshift. Studies show that such galaxies are significantly more compact than galaxies of comparable mass at low redshift (e.g., Daddi et al. 2005; Buitrago et al. 2008; Newman et al. 2012; Petty et al. 2013; Patel et al. 2017). The origin of this population of compact, massive galaxies at high- $z$ has been attributed to a phase of rapid gas accretion at early times (e.g., Dekel \& Burkert 2014), or major merger activity (e.g., Wuyts et al. 2010). The gas from these processes sinks toward the center of the galaxy, thus spurring bulge formation, heavily obscured star formation, and massive black hole growth. Subsequent growth via dry, stellar mergers is believed to be responsible for increasing galaxy sizes to produce the observed population seen today (Cappellari 2016). However, many unanswered questions remain, such as the redshift evolution of basic galaxies properties such as mass and size, the role of major mergers and the relative importance of different star formation quenching mechanisms in making massive galaxies passive (Wellons et al. 2015; Furlong et al. 2017; Pandya et al. 2017). Also unclear is why the star formation rate of a typical galaxy close to the knee in the galaxy mass function is much higher at redshifts $z \sim 1-3$ than in the current epoch (e.g., Rodighiero et al. 2014; Speagle et al. 2014).

Determining the physical mechanisms by which massive galaxies evolve into the objects we see today requires imaging high- $z$ galaxies on sub-kiloparsec scales. Imaging in the restframe optical/near-infrared, longward of the Balmer break, where the stellar population is dominated by the older stars, is particularly valuable compared to imaging the rest-frame UV, where the light is dominated by young stars and morphologies can be strongly affected by dust. Such imaging can be used to measure both the changing distribution of galaxy sizes as a 
function of redshift (e.g., van der Wel et al. 2014) and the frequency of interactions and mergers. Furthermore, by combining near-infrared imaging of the stellar light with high-resolution radio continuum imaging (which pinpoints the regions of star formation or nuclear activity in these systems) we can build up a much more complete picture of the nature of the galaxies. This is particularly valuable in dusty star-forming systems, where the peak of star formation activity may be offset from the peak of the visible stellar light (Rujopakarn et al. 2016, 2018).

Measurement of the morphologies of galaxies on subarcsecond scales has been dominated by observations made with the Hubble Space Telescope (HST). Adaptive Optics (AO) from the ground with large aperture telescopes is capable of delivering better image resolution at near-infrared wavelengths, but studies of field galaxies have, until recently, been restricted to small $\left(\approx 30^{\prime \prime}\right.$ diameter) patches of sky near individual guide stars (e.g., Glassman et al. 2002). The advent of MultiConjugate Adaptive Optics (MCAO) allows larger fields (up to $\approx 1.5$ in diameter) to be imaged by correcting multiple layers of the atmosphere, probed by multiple guide stars. This overcomes two limitations of conventional AO: (1) the limitation of the $\sim 30^{\prime \prime}$ radius isoplanatic patch over which correction from a single guide star is effective, and (2) the "cone effect" from laser guide stars, whereby the atmospheric turbulence probed by a single laser guide star is not the same as that from an arriving wavefront from a distant star (Tallon \& Foy 1990). The Gemini Multi-conjugate adaptive optics System (GeMS) on the Gemini South $8 \mathrm{~m}$ telescope (Neichel et al. 2014; Rigaut et al. 2014) uses a five-laser guide star and a natural guide star constellation of between one and three stars (with the best wide-field correction made with three stars) to achieve a consistent point-spread function (PSF) over a significantly wider $(\sim 1.5)$ field of view. Schirmer et al. (2015) demonstrated the effectiveness of this technique through imaging of the Hubble Frontier Fields MACS0416.1-2403 and Abell 2744, and Sweet et al. (2017) highlighted the technique through a study of cluster galaxies at $z=1$.

By obtaining subarcsecond-resolution imaging over a wide field of view, we can survey the sizes and morphologies of both the faint galaxy population and also extreme galaxies at high redshift, such as the hosts of active galactic nuclei (AGNs) and ultraluminous infrared galaxies (ULIRGs). As AO works well in the near-infrared $K$ band, at longer wavelengths than HST (whose longest wavelength filter on an operational instrument is in the $H$ band), $\mathrm{AO}$ is very suitable for investigating dusty galaxies detected in the infrared (e.g., Melbourne et al. 2011; Perna et al. 2015). Furthermore, the resolution available in these fields is sufficient to comfortably resolve the majority of the galaxy population over a wide range of redshifts.

Despite the recent advances in AO technology, the current use of GeMS is restricted to asterisms with stars brighter than $R \approx 15$ (depending on observing conditions), ideally consisting of three stars in an approximately equilateral triangle, and within an $\approx 2^{\prime}$ field of view (Neichel et al. 2014). Such asterisms are rare far from the Galactic Plane (we find $\sim 1 \mathrm{deg}^{2}$ ) and are even less commonly found in well-studied, small-area deep extragalactic fields, which are typically picked to avoid bright stars. Fortunately, the new generation of deep, wide-area $\left(\gg 1 \mathrm{deg}^{2}\right)$ extragalactic surveys, designed to study the evolution of galaxies over a wide range of environments, can also complement MCAO facilities by both containing suitable
Table 1

Gemini Observations Summary

\begin{tabular}{lcccc}
\hline \hline Field & $\begin{array}{c}\text { R.A. } \\
(\mathrm{J} 2000)\end{array}$ & $\begin{array}{c}\text { Decl. } \\
(\mathrm{J} 2000)\end{array}$ & Dates & $\begin{array}{c}\text { Time } \\
(\text { minutes })\end{array}$ \\
\hline ES1-C & $00: 35: 16.8$ & $-44: 01: 25$ & 2014 Dec 03 & 72.5 \\
XMM-C2 & $02: 27: 42.0$ & $-04: 33: 51$ & 2014 Dec 10-11 & 32.5 \\
CDFS-C & $03: 29: 13.8$ & $-28: 03: 15$ & 2015 Jan 04 & 65.0 \\
\hline
\end{tabular}

Note. A few observations were also obtained on other dates but could not be added into the final mosaic due to poor sky determination.

asterisms, and having the multiwavelength coverage needed to obtain photometric redshifts and star formation rates. The Spitzer Extragalactic Representative Volume Survey (SERVS; Mauduit et al. 2012) and associated VISTA Deep Extragalactic Observations (VIDEO) survey (Jarvis et al. 2013) provide $12 \mathrm{deg}^{2}$ of deep near-infrared observations in seven bands from $0.9-4.5 \mu \mathrm{m}$, enough area to find several such asterisms. We therefore took the opportunity to select asterisms in this survey suitable for observations with GeMS.

Deep, high-resolution imaging in the radio enabled by the new generation of wide-band correlators on radio interferometers has allowed a comparable transformation in the ability to image the faint radio emission from star-forming galaxies at $z \sim 1-3$. These observations have sufficient sensitivity to resolve emission on subarcsecond scales (e.g., Murphy et al. 2017). In cases where a radio excess above the maximum level expected from star formation is identified, these observations can distinguish emission due to star formation from that due to AGNs (e.g., Rujopakarn et al. 2016).

In this paper, we present results from a pilot study consisting of observations of the fields of three asterisms with GeMS and the Gemini South Adaptive Optics Imager (GSAOI) nearinfrared imager (Table 1). We combine the GeMs data with the multiwavelength data in the survey fields to obtain photometric redshifts and galaxy morphologies. We also present deep radio surveys in the two fields that contained the highest numbers of AGNs and Herschel sources. The radio surveys were made at arcsecond or better resolutions, with the aim of firmly identifying the host galaxies (or host galaxy components in a merging system) responsible for the bulk of the emission related to the AGNs and starbursts. We also discuss how ground-based MCAO observations can be used to complement the deeper observations that will be made with the James Webb Space Telescope (JWST). We assume a cosmology with $H_{0}=70 \mathrm{~km} \mathrm{~s}^{-1} \mathrm{Mpc}^{-1}, \Omega_{M}=0.3$ and $\Omega_{\Lambda}=0.7$.

\section{Observations}

\subsection{Observed Fields}

The three asterisms observed are drawn from three different SERVS fields: ELAIS-S1 (centered on R.A.(J2000) = 00:37:48, decl.(J2000) $=-44: 00)$, XMM-LSS (centered on R.A.(J2000) $=$ 02:20:00, decl.(J2000) $=-04: 48)$, and CDFS (centered on R.A.(J2000) = 03:32:19, decl.(J2000) $=-28: 06)$. All SERVS fields were observed to the same depth (20 minutes) during the post-cryogenic Spitzer mission in the 3.6 and $4.5 \mu \mathrm{m}$ bands, with the observational scheme described in Mauduit et al. (2012) reaching a $5 \sigma$ depth of $\approx 2 \mu \mathrm{Jy}$ in each band.

These fields have been the subject of extensive multiwavelength studies, and include data from the optical through 
Table 2

Multi-band Infrared Data Common to All Fields

\begin{tabular}{|c|c|c|c|}
\hline Bands & Depth $^{\mathrm{a}}$ & Survey $^{\mathrm{b}}$ & References \\
\hline$Z, Y^{\mathrm{a}}, J, H, K_{\mathrm{s}}$ & $26.3,25.2,25.1,24.6,24.1$ & VIDEO DR4 & Jarvis et al. (2013) \\
\hline $3.6,4.5 \mu \mathrm{m}$ & $23.7,23.7$ & SERVS & Mauduit et al. (2012) \\
\hline $5.8,8.0,24,70 \mu \mathrm{m}$ & $20.3,20.5,18.6,16.3$ & SWIRE & Lonsdale et al. (2003) \\
\hline $250,350 \mu \mathrm{m}$ & $16.3,16.3$ & HerMES & Oliver et al. (2012) \\
\hline
\end{tabular}

Notes.

a All depths are given at approximately the $3 \sigma$ level (AB mag).

b The CDFS-C field did not have $Y$-band data available from the VIDEO survey at the time of writing.

the infrared. Of particular relevance to this paper is the HerMES survey (Oliver et al. 2012) with the Herschel telescope (Pilbratt et al. 2010). The Herschel/SPIRE observations in the three fields differed in exposure time; however, they are all confusion-limited at noise levels of $\approx 6 \mathrm{mJy}$ at 250 and $350 \mu \mathrm{m}$, and $\approx 10 \mathrm{mJy}$ at $500 \mu \mathrm{m}$. In all three fields we used near-infrared $Z, Y, J, H$, and $K_{\mathrm{s}}$ data from the VIDEO survey (Jarvis et al. 2013) and data from the SIRTF Wide-area Infrared Extragalactic Legacy Survey (SWIRE; Lonsdale et al. 2003 ) in the $5.8,8.0,24$, and $70 \mu \mathrm{m}$ bands.

The source of the optical data depended on the field, in ES1 we used data from our own $i$-band survey (described in Section 2.4 below) and the preliminary release of the Dark Energy Survey (DES; Dark Energy Survey Collaboration et al. 2016), in XMM-LSS we used data from the Canada-FranceHawaii Telescope Legacy Survey (CFHTLS; Gwyn 2012), and in CDFS we used ancillary optical data from the SWIRE survey. Our use of archival data sets, including approximate depths, is summarized in Tables 2 and 3.

\subsection{Gemini MCAO Observations}

We selected asterisms in the SERVS fields that were as close as possible to the ideal equilateral triangle configuration that yields a near-uniform PSF across the field (Figures 1-3, with the PSFs shown in Figure 4). The three fields were observed in Gemini program GS-2013B-Q14. ES1-C is in the SERVS ELAIS-S1 field, XMM-C2 is in the XMM-LSS field, and CDFS-C is in the CDFS field. Observations are listed in Table 1 and further details of the observational conditions for AO are listed in Table 4. Typical Strehl ratios (the ratio of the measured PSF peak to that obtained considering only diffraction; see Section 3.9) in the $K$ band for the ES1-C and CDFS-C observations were $10 \%-15 \%$, comparable to the values seen in commissioning (Neichel et al. 2014). The conditions for the XMM-C2 observations were significantly worse, with a Strehl ratio $\approx 5 \%$. Nevertheless the observations resulted in a better FWHM than is obtainable from non-AO observations. Each field is $\approx 2 \operatorname{arcmin}^{2}$ in area.

We chose the $K$ band since it is both likely to have the highest Strehl ratio, and it samples the rest-frame optical at $z \sim 3$, yet cannot be observed with HST. Observations were obtained through the $K^{\prime}$ filter (central wavelength $2.11 \mu \mathrm{m}$ ), which we judged to be the best compromise between bandwidth and low thermal noise. We used 150-second exposures, dithering up to $8^{\prime \prime}$ between each observation in a random pattern.
Table 3

Optical Data

\begin{tabular}{lcc}
\hline \hline Field & Optical Data Sources & Depth $^{\mathrm{a}}$ \\
\hline ES1-C & DES $g$ and $r$ band; CTIO & $g \approx 25.0, r \approx 25.0, i \approx 25.6$ \\
& $i$ band (this paper) & $u \approx 26.4, g \approx 26.1, r \approx 25.6$, \\
XMM-C2 & CFHTLS D1; $u, g, r, i, z$ & $i \approx 25.3, z \approx 25.0$ \\
& & $r \approx 25.0, g \approx 25.0, u \approx 25.5$ \\
CDFS-C & SWIRE ancillary optical & $r \approx$ \\
\hline
\end{tabular}

Note.

a The CFHTLS depths are based on the $80 \%$ completeness values from the T0007 Final Release document (http://terapix.iap.fr/cplt/T0007/doc/T0007doc.pdf).

\subsection{Radio Observations}

The ES1-C field was observed in four runs with the Australia Telescope Compact Array (ATCA) at 8.4 GHz, 2016 March 10-13. We obtained $56 \mathrm{hr}$ of data. The XMM-C2 field was observed in a single pointing with the VLA in A-configuration as part of project VLA/14A-353 over the frequency range 8-12 GHz. Twelve executions of a single $1 \mathrm{hr}$ scheduling block were observed in filler time, each spending 12.7 minutes on the XMM-C2 field, for a total time on-source of 152 minutes.

\subsection{Optical Observations in ES1}

The ES1 field of SERVS was observed with the Cerro Tololo Inter-American Observatory (CTIO) Mosaic II camera in the $i$ band in two runs, one on 2009 November 25, and the other from 2010 September 30 to 2010 October 4. Twelve pointings were observed so as to cover the whole SERVS ES1 field (with the ES1-C field observed in the 2010 observations).

\section{Data Reduction and Analysis}

\subsection{Gemini Image Reduction}

The images were run through the standard Gemini GSAOI software steps. Dark subtraction and flat-fielding were applied using appropriate observatory calibration files found in the Gemini archive. The FITS files were then split up into individual detector arrays. The data were then used to form a median background image, which was then subtracted, and the images were then shifted (using a bright, but unsaturated object as a reference) and averaged together, with the highest and lowest values for each pixel being rejected from the stack. Array 2 was found to suffer from the reset anomaly described in Schirmer et al. (2015). A similar solution was applied whereby the median dark from the other images in the stack was scaled before being subtracted from the first frame. 


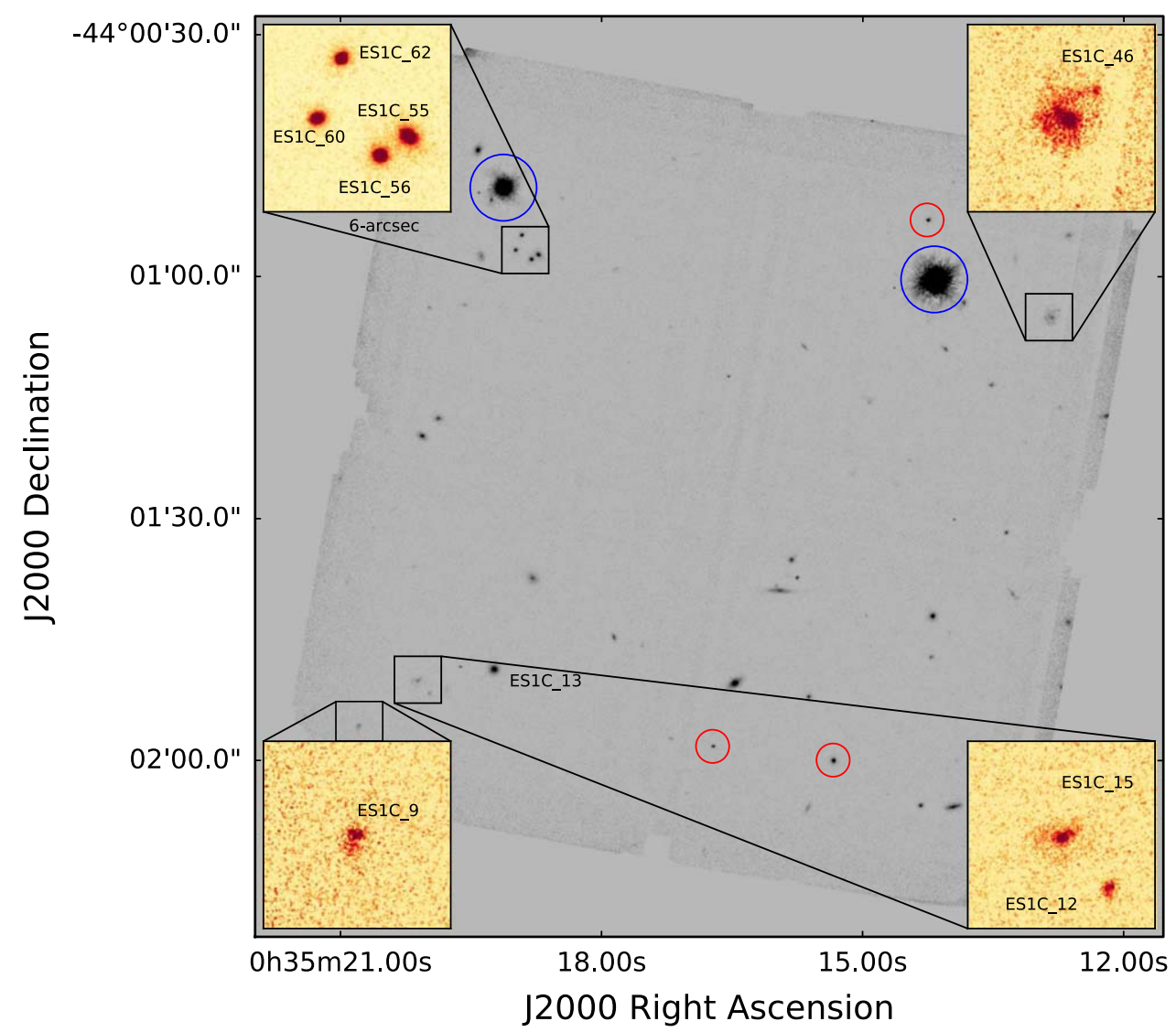

Figure 1. GSAOI image of the ES1-C field. Objects of interest due to their detection at other wavebands discussed in Section 4.2, or with noteworthy morphologies (Section 4.3), are shown as insets, each measuring $6^{\prime \prime}$ per side. The red circles indicate the stars used to determine the PSF in the field, and the blue circles show those used as natural guide stars for the adaptive optics system (one is off the image). Note that the guide stars were saturated, so they cannot be used for PSF determination.

Table 4

Gemini Adaptive Optics and PSF Parameters

\begin{tabular}{|c|c|c|c|c|}
\hline $\begin{array}{l}\text { Field } \\
\text { (1) }\end{array}$ & $\begin{array}{c}\text { Strehl } \\
\text { Ratio } \\
\text { (2) }\end{array}$ & $\begin{array}{c}\theta_{\mathrm{FWHM}} \\
\text { (mas) } \\
(3)\end{array}$ & $\begin{array}{c}\text { PSF } \\
\text { Model FWHM } \\
\text { pixels } \\
\text { (4) }\end{array}$ & $\begin{array}{l}\text { PSF Model } \\
\text { Weights } \\
\text { (5) }\end{array}$ \\
\hline ES1-C & $10 \%$ & 94 & $1.88,4.58,15.2$ & $0.14,0.54,0.32$ \\
\hline XMM-C2 & $5 \%$ & 155 & $1.88,4.58,15.2$ & $0.14,0.54,0.32$ \\
\hline CDFS-C & $16 \%$ & 70 & $\begin{array}{l}1.73,3.96 \\
10.0,22.26\end{array}$ & $\begin{array}{c}0.22,0.34 \\
0.19,0.24\end{array}$ \\
\hline
\end{tabular}

Note. Column (1): field name. Column (2): the PSF Strehl ratio (the ratio of the peak of the measured PSF to the theoretical PSF); see the text. Column (3): FWHM of the observed PSF. Column (4): FWHM of the mixture of circular Gaussians used to form the PSF model after the weights are applied. Note that the PSF model from the ES1-C field was used for XMM-C2 due to the relatively poor quality of the observations in that field. Column (5): weights of each component of the mixture of circular Gaussians used to form the PSF model. The weights are normalized to unity.

Sources in the reduced image for each array were then matched to detections in the VIDEO survey (Jarvis et al. 2013). The pixel scale of the image given in the header ( $0.0194 \mathrm{arcsec} / \mathrm{pixel})$ was checked and was found to be correct within the uncertainty of the VIDEO positions; however, small corrections were applied to the rotation and center of each array to align them with the VIDEO reference frame, using about 10 objects per detector to perform the fitting. Within the scatter in our reference astrometry of $\approx 0 . " 05$, no evidence of nonlinear distortions was found.

As a check, we also fit the ES1-C image (which has the highest source density) for distortions using SCAMP (Bertin 2006) with the VIDEO positions as a reference and confirmed that any remaining distortion is $<2 \%$ in linear scale across the frame. This results in a largest shift of $0 . " 5$, consistent with that found by Schirmer et al. (2015). As we are not using the data for high-precision astrometry or photometric applications, we thus decided not to correct the frames for distortion. The Montage software (Berriman et al. 2003) was then used to combine the four arrays back into a single image. Flux calibration was obtained by scaling to the VIDEO catalog for isolated objects. The observations in the deepest two fields (ES1-C and CDFS-C) reach a $5 \sigma$ depth of $\operatorname{mag}_{\mathrm{AB}} \approx 24.6$ for point sources. The XMM-C2 data, which were observed for a shorter time, reach a $5 \sigma$ depth of $\approx 24.0$. Grayscale images of the fields are shown in Figures $1-3$.

\subsection{Radio Data Processing}

Standard data reduction of the ATCA data was performed in MIRIAD (Sault et al. 1995). Phase-only self-calibration was applied, and images were made with natural and uniform weighting. In the naturally weighted map we obtained an rms noise of $1.8 \mu \mathrm{Jy}$ beam $^{-1}$ with a synthesized beam size of 2 ". $1 \times 1$ ". 9 at a position angle (PA) of $0^{\circ}$. The uniformly 


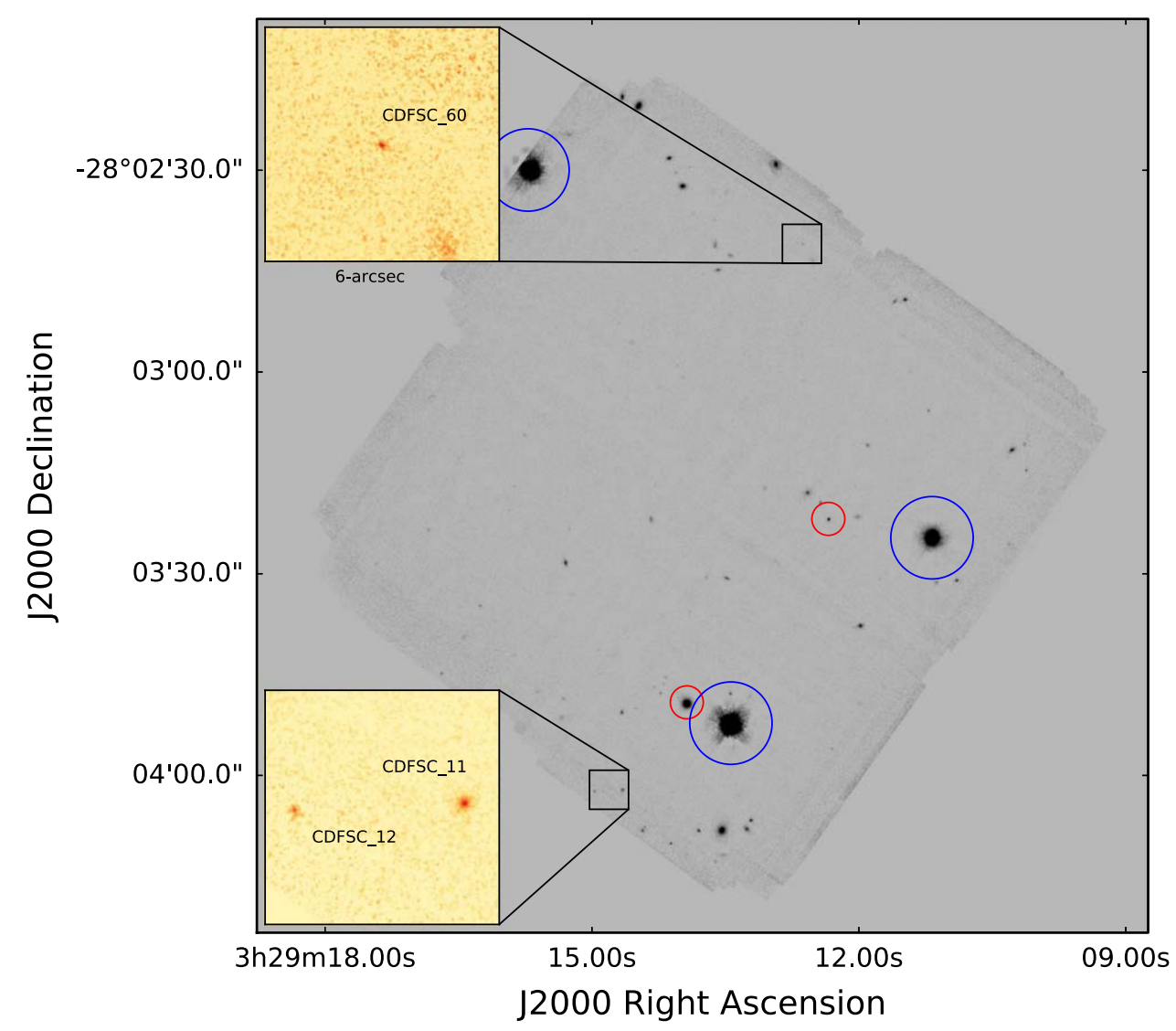

Figure 2. GSAOI image of the CDFS-C field. Three $z \sim 3$ compact galaxies (Section 4.3 ) are shown in the insets, each measuring $6 "$ per side. The red circles indicate the stars used to determine the PSF in the field, and the blue circles show those used as natural guide stars for the adaptive optics system. Note that the guide stars were saturated, so they cannot be used for PSF determination.

weighted map has an rms noise of $2.3 \mu \mathrm{Jy}^{\text {beam }^{-1}}$ with a synthesized beam size of 1 !"9 $\times 1{ }^{\prime \prime} 2$, also at $\mathrm{PA}=0^{\circ}$.

The VLA data were reduced in CASA (McMullin et al. 2007) following standard procedures. No self-calibration was applied. The synthesized beam size of our final image was 0 ! $21 \times 0$ !" 16 (naturally weighted) at PA $=48^{\circ}$. The image reaches an rms noise of $0.45 \mu \mathrm{Jy}$ beam $^{-1}$.

\subsection{Optical Data Processing}

Data from the 2010 CTIO run in ES1 were reduced at the Cambridge Astronomical Survey Unit; those from 2009 were reduced using the IRAF mscred package. The data were photometrically calibrated by cross-matching to DES (Dark Energy Survey Collaboration et al. 2016) data available from the National Optical Astronomical Observatory archive. The resulting image reaches an average $5 \sigma$ depth of $i \approx$ $25 \mathrm{mag}$.

\subsection{Point-spread Functions}

We show the PSFs of stars in the ES1-C and CDFS-C fields in Figure 4 using the stars circled in red in Figures 1 and 2. The small number of unsaturated stars makes it difficult to assess the level of PSF variation across the field, though our measurements suggest it is small, $\sim 10 \%$ of the FWHM. We estimated the Strehl ratio for our observations by comparing the peak of a PSF model evaluated at the same pixel phase as our brightest PSF in each field to the observed peak value (in a 3 !" $8 \times 3$ !" 8 box). We used a simple obscured aperture model for the PSF:

$$
I(\nu)=\frac{1}{\left(1-f^{2}\right)^{2}}\left[\frac{2 J_{1}(\nu)}{\nu}-f^{2} \frac{2 J_{1}(f \nu)}{f \nu}\right]^{2},
$$

where $\nu=\theta \pi D / \lambda, \theta$ is the off-axis angle, $D=8.1 \mathrm{~m}$ is the effective diameter of the Gemini mirror, $f=0.123$ is the fractional radial obscuration of the aperture caused by the central hole (values of $D$ and $f$ are from Turri et al. 2017), and $J_{1}$ is the first-order Bessel function.

In order to use the Tractor photometry code (Section 3.5) we modeled the PSFs as sums of up to four circular Gaussians of different FWHM, modeling the stars in each field separately and averaging the results for each field. The Gaussians for a given star were all centered on a single position, which was allowed to vary during the fitting. The best fit was determined by minimizing the $\chi$-squared of the residuals. The Strehl ratio, FWHM, and the weights of the best-fitting Gaussian components used in each field are listed in Table 4. In ES1-C the PSFs were well fit by three circular Gausssians, and in CDFS-C four Gaussians were needed to capture the extended wings of the PSF. The XMM-C2 field was taken in significantly poorer conditions than the other two fields, and with less integration 


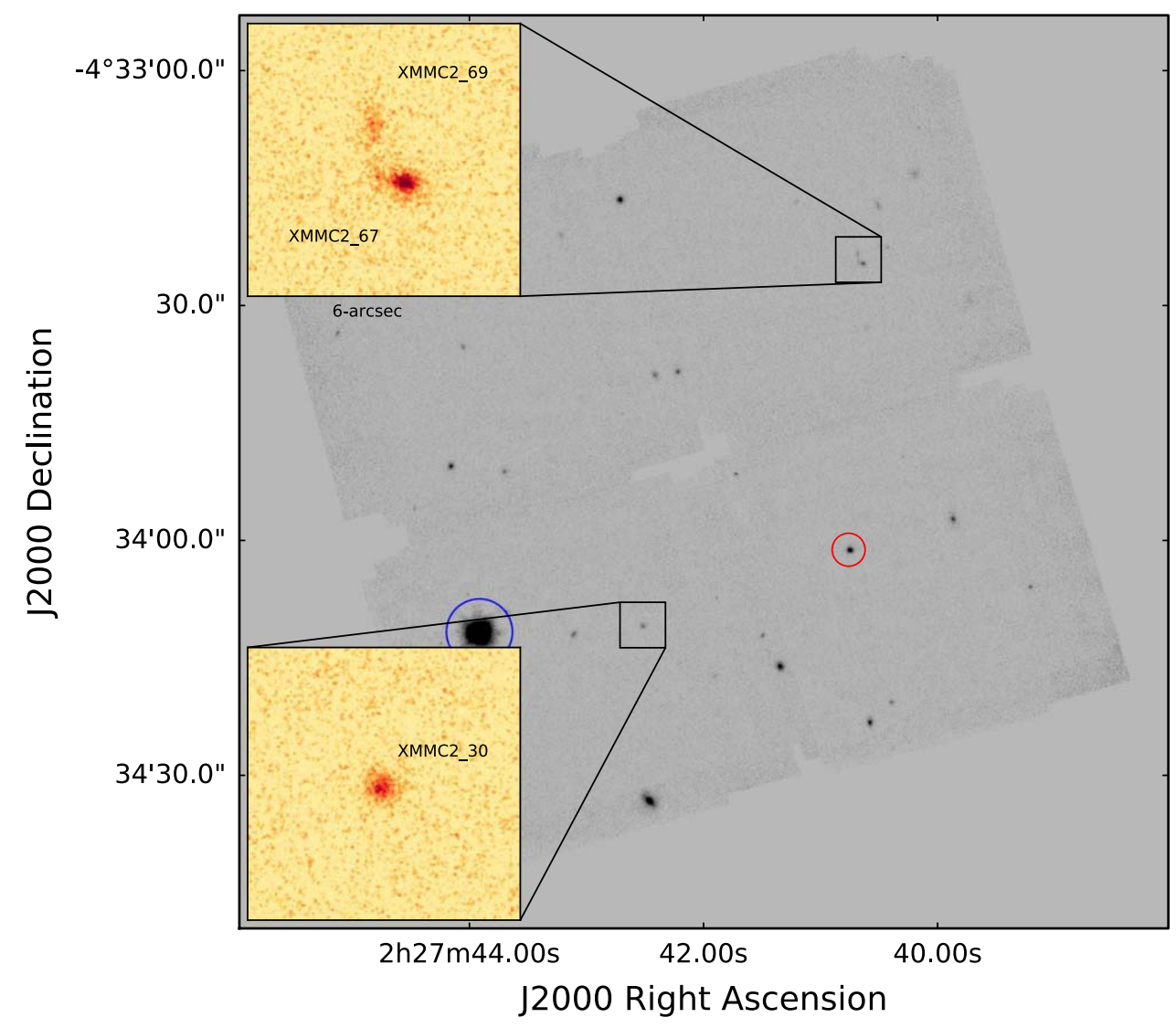

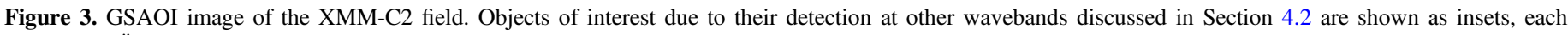

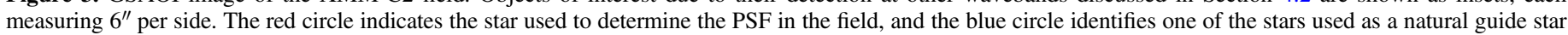
for the adaptive optics system (the other two are off the image). Note that the guide star was saturated, so it cannot be used for PSF determination.
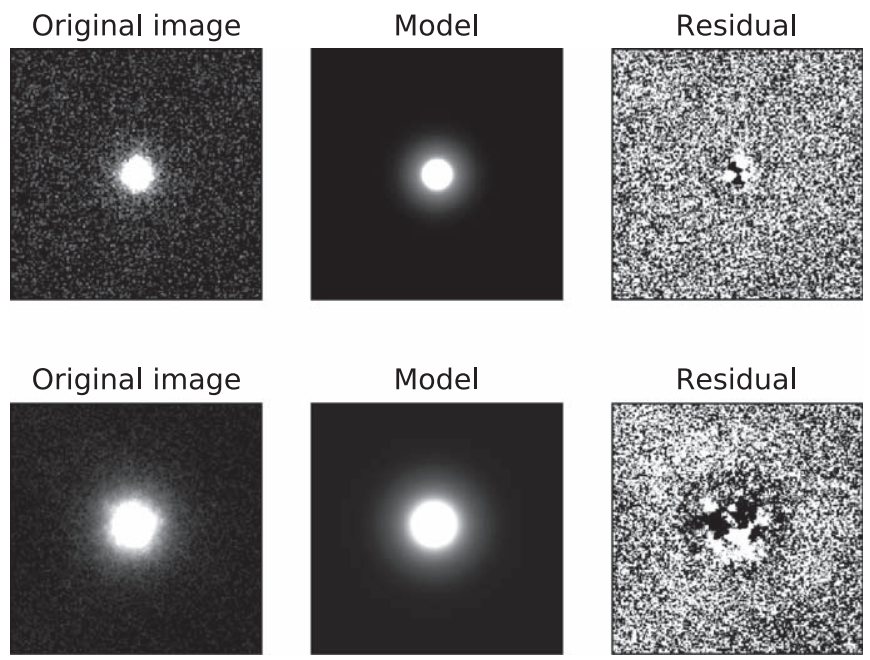

Figure 4. PSFs used in the Tractor fitting for ES1-C (top three images) and CDFS-C (bottom three images). From left to right, the panels show one of the PSF stars, the PSF model, and the residual. The stars used to estimate the PSF are shown in Figures 1 and 2. The cutouts are $4^{\prime \prime}$ on a side; in the top figure the grayscale range is from 0 to 100 counts in the left two images, and -10 to 10 in the residual image; and in the bottom (where a brighter PSF star was available) the ranges are from 0 to 500 and -10 to 10 . Note that the data quality in the XMM-C2 field was low compared to that in the other two fields, so we used the ES1-C PSF for the analyses of those data.

time, rendering an accurate PSF fitting not worthwhile, so we adopted the same PSF parameters as the ES1-C field for the purposes of the Tractor fitting.

\subsection{Optical to Near-infrared Photometry}

We used the Tractor (Hogg \& Lang 2013; Lang et al. 2016) to perform photometry matched between the high-resolution Gemini images and the lower-resolution images from SERVS, VIDEO, CFHTLS, and other optical data (Tables 2 and 3). The Tractor provides a convenient method of fitting modelbased galaxy photometry without the need to re-sample images with widely differing pixel scales (Nyland et al. 2017). Sources in the Gemini data were fit either as point sources, or if resolved, with either exponential disk or de Vaucouleurs profiles (only a handful of the galaxies had high enough signal-to-noise ratios to reliably justify fitting a Sérsic profile using the Tractor, so we did not perform fits to Sérsic profiles). PSFs in all bands were modeled as mixtures of circular Gaussians following the strategy described for the Gemini data in Section 3.4.

An initial catalog was compiled by running SExtractor (Bertin \& Arnouts 1996) on the Gemini data. This was then used to supply initial guesses for the fit parameters in our Python implementation of the Tractor. A total of 165 objects were fit: 64 in ES1-C, 57 in CDFS-C, and 44 in the XMM-C2 field. We looped through our input catalog on a source-bysource basis, extracting a $10^{\prime \prime}$ cutout image at each source location. For each source in our input catalog, we fit either a point source or resolved surface brightness profile model. Point-source models were fit for sources with a SExtractor stellaricity parameter $<0.1$ or SExtractor size estimate $<0$ ". 05 . Sources not meeting these criteria were modeled with either an exponential disk or de Vaucouleurs profile. All sources were fit 
Table 5

Optical through Near-infrared Tractor Photometry

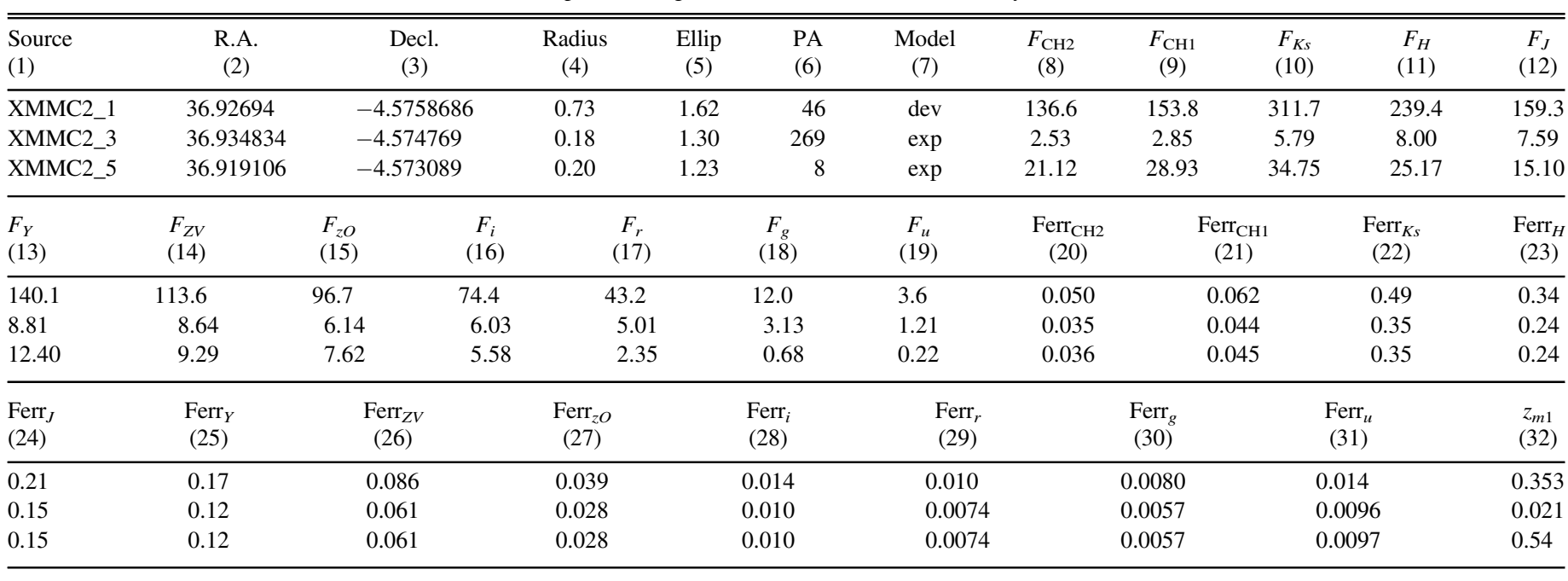

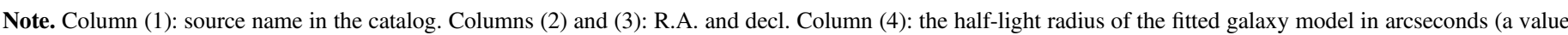

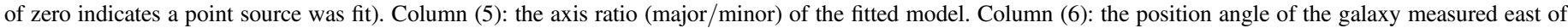

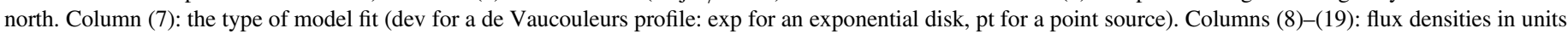

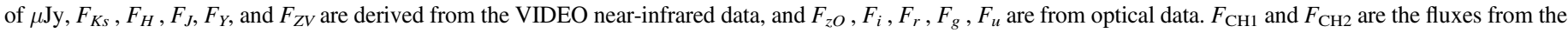

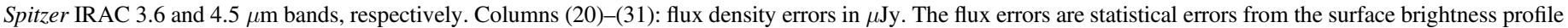
fits performed during the Tractor photometry and do not include calibration errors. Column (32): the best fit photometric redshift from EAZY.

(This table is available in its entirety in machine-readable form.)

for position and flux, and resolved sources for ellipticity and PA. ${ }^{19}$

The Tractor operates by convolving the source model with the PSF model at each band and then performing an optimization to determine the image flux, position, and shape properties. The code was run twice, once fitting exponential disk models to resolved sources and once fitting de Vaucouleurs models, and the best-fit (lower reduced chisquared) model was chosen for each object. Objects with radii consistent with zero from either run were refit with pointsource models. The final photometry is given in Table 5. Further details on our implementation of the Tractor image modeling code may be found in Nyland et al. (2017).

\subsection{Mid-infrared to Far-infrared Photometry}

There are several Herschel sources in the ES1-C and XMMC2 fields detected in the HerMES survey (Oliver et al. 2012) by the SPIRE instrument at 250 and $350 \mu \mathrm{m}$ (the CDFS-C field lacks Herschel detections). We used prior data from the SWIRE MIPS $24 \mu \mathrm{m}$ survey and our deep radio imaging to assess whether star formation or AGN emission dominates the infrared flux from each Herschel source (Table 7). We then used the Tractor to simultaneously fit point-source models to the SWIRE 5.8, 8, and $24 \mu \mathrm{m}$ data and the HerMES 250 and $350 \mu \mathrm{m}$ data at the position of every optical/near-infrared galaxy with a mid-infrared or radio detection that lay within the contours of the Herschel source. The Herschel sources also appear in the HerMES DR4 catalogs (which use positional priors from Spitzer $24 \mu \mathrm{m}$ observations; Oliver et al. 2012, using a predecessor to the XID+ code of Hurley et al. 2017). We have normalized the sums of the component Tractor fluxes

\footnotetext{
19 We note this differs from the procedure described in Nyland et al. (2017), where the the shape and position parameters were held fixed to improve speed and stability, and the Tractor was used only to fit the fluxes of the objects.
}

to the sums of the components of the same Herschel detections in the HerMES DR4 catalog to ensure a consistent flux density scale. In some cases our attribution of fluxes among components has differed from that in the HerMES catalog; those cases are detailed in Section 4.2.

\subsection{Photometric Redshifts and Stellar Masses}

Photometric redshifts were estimated using the EAZY software (Brammer et al. 2008). They were checked against photometric redshifts from the SERVS photometric redshift catalog (J. Pforr et al. 2018, in preparation) for objects that are detected in the SERVS catalogs. In addition to the redshifts, the EAZY code was used to calculate the rest-frame $U-V$ and $V-J$ colors used in Figure 5.

Stellar masses $\left(M_{*}\right)$ were estimated by performing spectrophotometric fitting of stellar population models with the HyperZ code (Bolzonella et al. 2000) and using the ancillary scripts for $M_{*}$ calculation as in Daddi et al. (2005) and Maraston et al. (2006). As in these papers, data were fitted using a comprehensive suite of models (from Maraston 2005) for a variety of star formation histories, spanning from single bursts to constant star formation rates, each calculated for 221 ages from 0.001 to $15,000 \mathrm{Myr}$, and four metallicities below, at, and above solar $([\mathrm{Z} / \mathrm{H}]=-1.35,-0.33,0.0$, and 0.35$)$. Calculations were performed assuming no reddening, a Calzetti reddening law (Calzetti et al. 1994), and a reddening law typical of the Small Magellanic Cloud (SMC). The noreddening option is useful to check how much the derived stellar mass is affected by age-dust degeneracies, which may lead to an underestimate of $M^{*}$ for star-forming galaxies (see Pforr et al. 2012). This is known as the "iceberg effect" (Maraston et al. 2010), which occurs when, by fitting the youngest stellar populations with reddening, the estimated stellar mass is closer to the mass involved in the burst (the tip 

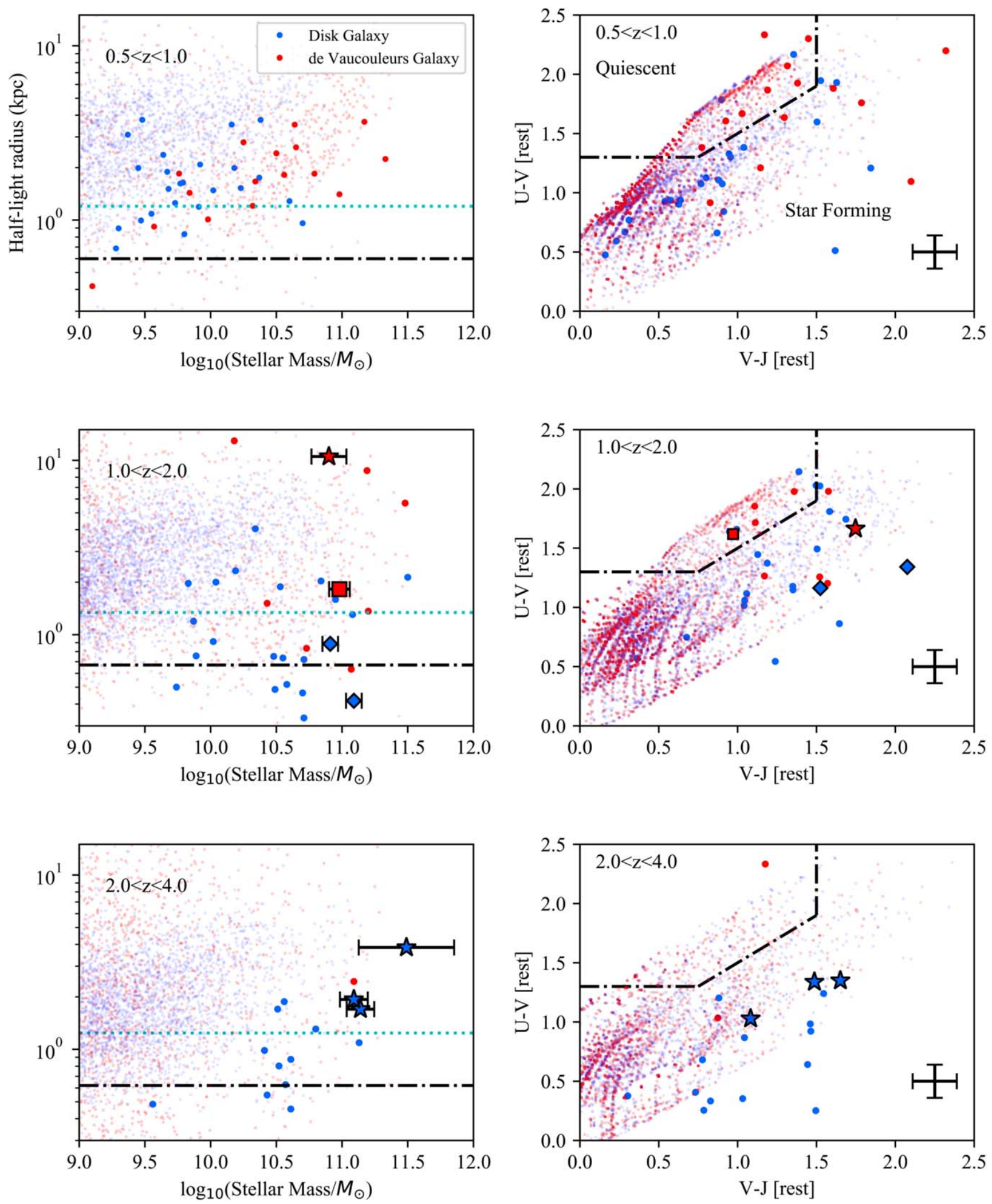

Figure 5. Sizes, colors, and stellar masses of the galaxies identified in our study, split into three redshift bins. Galaxies best-fit by disks are shown in blue; those best fit by de Vaucouleurs profiles are shown in red. Objects from the CANDELS-COSMOS field (van der Wel et al. 2012; Nayyeri et al. 2017) are shown for comparison as faint dots, where we have colored objects fit with Sérsic indices $<2.5$ in blue and those fit with Sérsic indices $>2.5$ in red. Left column: half-light radius vs stellar mass; right column: rest-frame $U-V$ color vs. rest-frame $V-J$ color. Radio-quiet AGNs are indicated with diamonds, radio-loud AGNs are indicated with squares, and starbursts are indicated with stars. In the left column, the black dotted-dashed line indicates the resolution of the Gemini images and the cyan dotted line indicates the resolution of $H S T$ /WF3 in the F160W filter. In the right column, the dotted-dashed line indicates the divide between quiescent (upper left) and star-forming (lower right) galaxies, and the typical error bar is shown on the bottom right. 

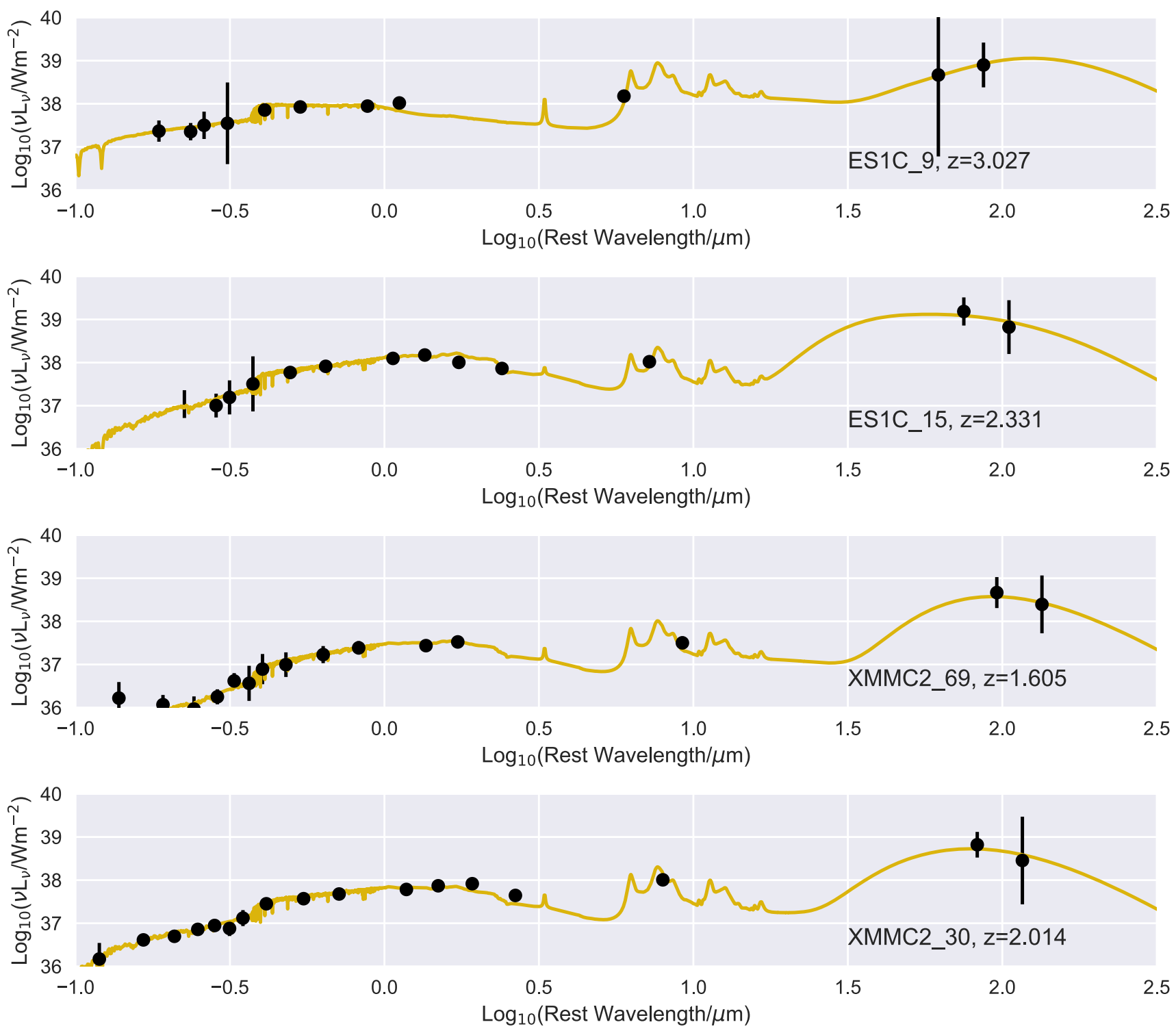

Figure 6. SEDs of galaxies dominated by star formation. The gold solid lines are the fits from MAGPHYS.

of the iceberg) rather than to the total one. The SMC law is included in addition to the Calzetti law, as it is found to give good results in high- $z$, passive galaxies (Maraston et al. 2006; Kriek \& Conroy 2013). In the calculations, the EAZY photometric redshifts were assumed for constraining the fits.

\subsection{SED Fitting}

We classified the sources detected in the Herschel images as pure starbursts or composite starbursts+AGN based on their mid-infrared colors (e.g., Lacy et al. 2004). In addition, we identify one radio-loud AGN that does not have significant farinfrared flux. The purely star-forming galaxies were fit using the MAGPHYS code (da Cunha et al. 2008), which models the three emission components of the ISM (polycyclic aromatic hydrocarbons, warm dust, and a cooler dust component) and UV emission from starlight in a self-consistent way (Figure 6). The reduced $\chi^{2}$ values of the fits vary from 1.5 to 8.2 . All Herschel sources have infrared luminosities $L_{\mathrm{IR}}>10^{12} L_{\odot}$ and thus qualify as ULIRGs.
For relatively bright objects in the mid-infrared consistent with mixed AGNs and star formation activity, we used the composite AGN/starburst templates from Kirkpatrick et al. (2015) to estimate the star formation rates, as shown in Figure 7. We selected the closest template to the SEDs of our AGNs, the "Composite-2" SED (with an AGN contribution to the IR luminosity of $60 \%$ ). Although the mid-infrared excess from the AGN dust is larger in our objects than the template (suggesting a larger AGN contribution), the far-infrared SED matches well. ${ }^{20} \mathrm{We}$ also provide estimates of star formation rates based on both the far-infrared emission and the radio emission using Equations (4) and (6) from Bell (2003). The results are given in Table 8 and details about each object are given in Section 4.2 .

\footnotetext{
${ }^{20}$ We note that the Kirkpatrick et al. (2015) templates have too low of a farinfrared to mid-infrared flux ratio to fit our two most actively starbursting galaxies, probably because the sample used to make them was selected in the mid-infrared.
} 

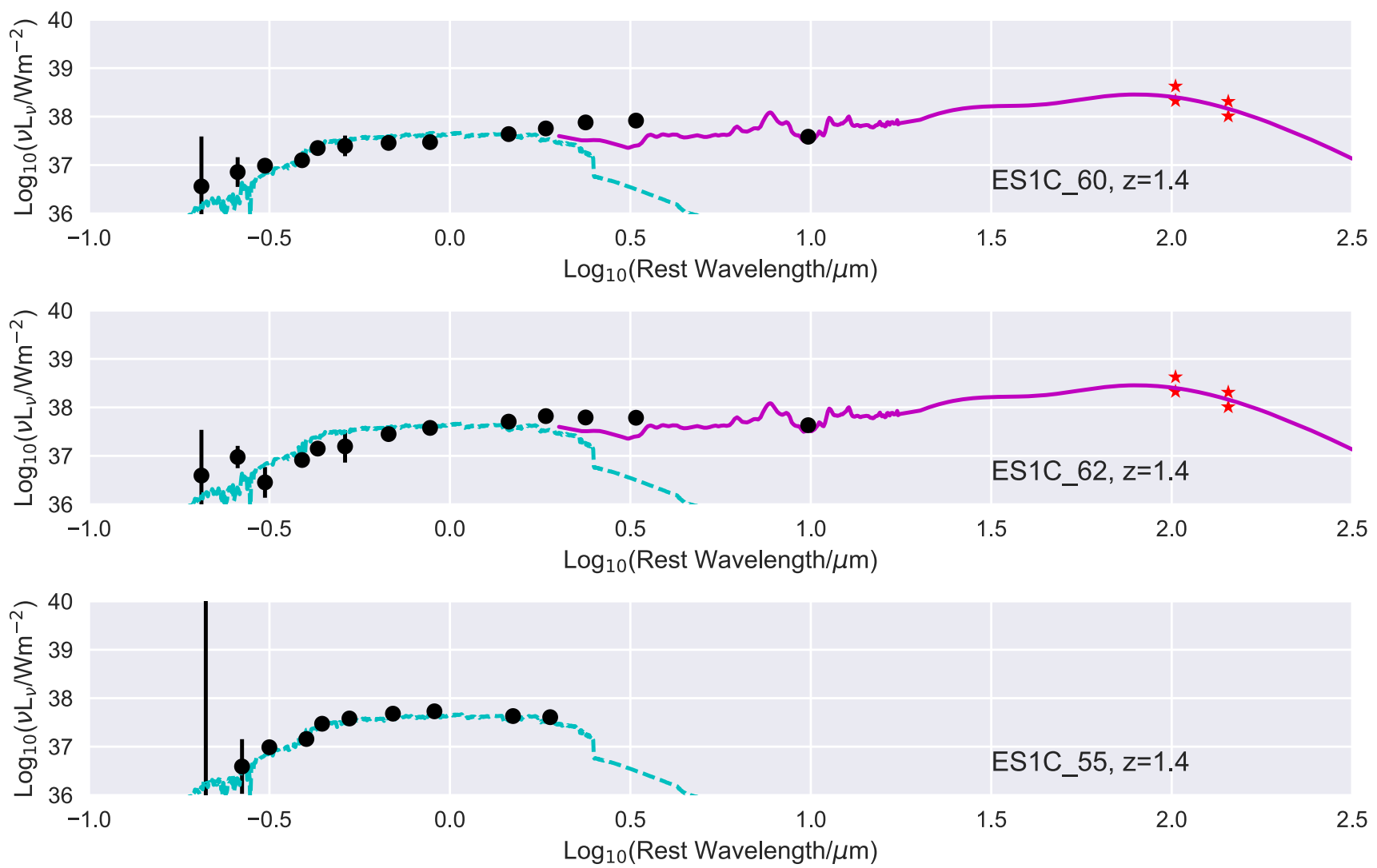

Figure 7. SEDs of the candidate triple-AGN in the ES1-C field. The cyan dashed lines are a single $1 \mathrm{Gyr}$ stellar population from Maraston (2005) and the solid magenta lines are the Composite-2 SED from Kirkpatrick et al. (2015). The Composite-2 SED was not fit to the radio galaxy (ES1C_55), as it lacks detectable midinfrared emission. The two sets of stars in the top two panels showing ES1C_60 and ES1C_62 indicate the total fluxes in the blended Herschel data (upper stars) and a $50 \%$ attribution to each object (lower stars).

\subsection{The Radio-Far-infrared Correlation}

The tight radio-far-infrared correlation extends over three orders of magnitude in normal star-forming galaxies (Helou et al. 1985). First seen locally, it is now known to extend out to at least $z \sim 2$ (e.g., Ivison et al. 2010; Mao et al. 2011; Magnelli et al. 2015; Delhaize et al. 2017), though the nature of the redshift evolution of the relation remains a subject of debate. Here, we use the radio-infrared correlation as a diagnostic tool for identifying radio AGNs based on the presence of a significant excess of radio emission compared to the level expected purely from star formation.

We use our new ATCA and VLA radio continuum data and the estimates of total infrared luminosity from our SED modeling $\left(S_{\mathrm{IR}}\right)$ to estimate the logarithmic infrared to radio flux ratio, $q_{\mathrm{IR}}=\log _{10}\left[\left(S_{\mathrm{IR}} / 3.75 \times 10^{12} \mathrm{~W} \mathrm{~m}^{-2}\right) /\left(S_{1.4 \mathrm{GHz}} / \mathrm{W} \mathrm{Hz}^{-1} \mathrm{~m}^{-2}\right)\right]$, following Ivison et al. (2010). $S_{1.4 \mathrm{GHz}}$ is calculated in the restframe assuming a radio spectral index ${ }^{21}$ of $\alpha=-0.8$. We provide the $q_{\mathrm{IR}}$ values for our sources in Table 7 . The $q_{\mathrm{IR}}$ values indicate that most of the Herschel sources lie close to the typical value for star-forming galaxies of $q_{\mathrm{IR}} \approx 2.4$, certainly within the $\pm \approx 0.4 \mathrm{dex}$ scatter seen by Ivison et al. (2010) for Herschel sources. As expected, the radio-quiet AGN pair ES1C_60/ES1C_62 lies above the relation $\left(q_{\mathrm{IR}}=3.1\right)$, though when the infrared flux is reduced by $60 \%$ to allow for the AGN contribution, it falls within the scatter expected for normal star-forming galaxies.

\footnotetext{
${ }^{21}$ We follow the convention for radio spectral index $(\alpha)$ of $S \sim \nu^{\alpha}$, where $S$ is the flux, and $\nu$ is the frequency.
}

\section{Results}

\subsection{Galaxies in the Field}

Figure 5 shows the sizes, colors, and stellar masses of the galaxies considered in our study for three different redshift ranges. The redshift bins were picked to roughly divide the study into epochs of later galaxy evolution $(0.5<z<1.0-$ the lower limit was picked, as there are very few $z<0.5$ galaxies with masses in the same range as the higher- $z$ objects), peak galaxy formation $(1.0<z<2.0)$, and early galaxy formation $(2<z<4)$. (There are too few $z>4$ objects with reliable photo-zs to explore higher in redshift.) Galaxy size evolution is closely tied to merger activity, as dry mergers can "puff up" small galaxies (e.g., Bluck et al. 2012; Newman et al. 2012), and estimates of the galaxy size distribution as a function of redshift are thus important for our understanding of the importance of mergers in galaxy evolution. As seen in prior studies with HST (e.g., van der Wel et al. 2014; Shibuya et al. 2015; Allen et al. 2017), there is strong evidence for size evolution at a given stellar mass, particularly for galaxies with masses $<10^{11} M_{\odot}$. The right column of Figure 5 shows the $U-V$ versus $V-J$ rest-frame color-color diagram that Whitaker et al. (2011) use to separate star-forming galaxies from quiescent galaxies. This figure shows that our morphological classifications are broadly in line with the expectations from this plot, namely that the quiescent galaxies include a large fraction with de Vaucouleurs profiles at all redshifts, 


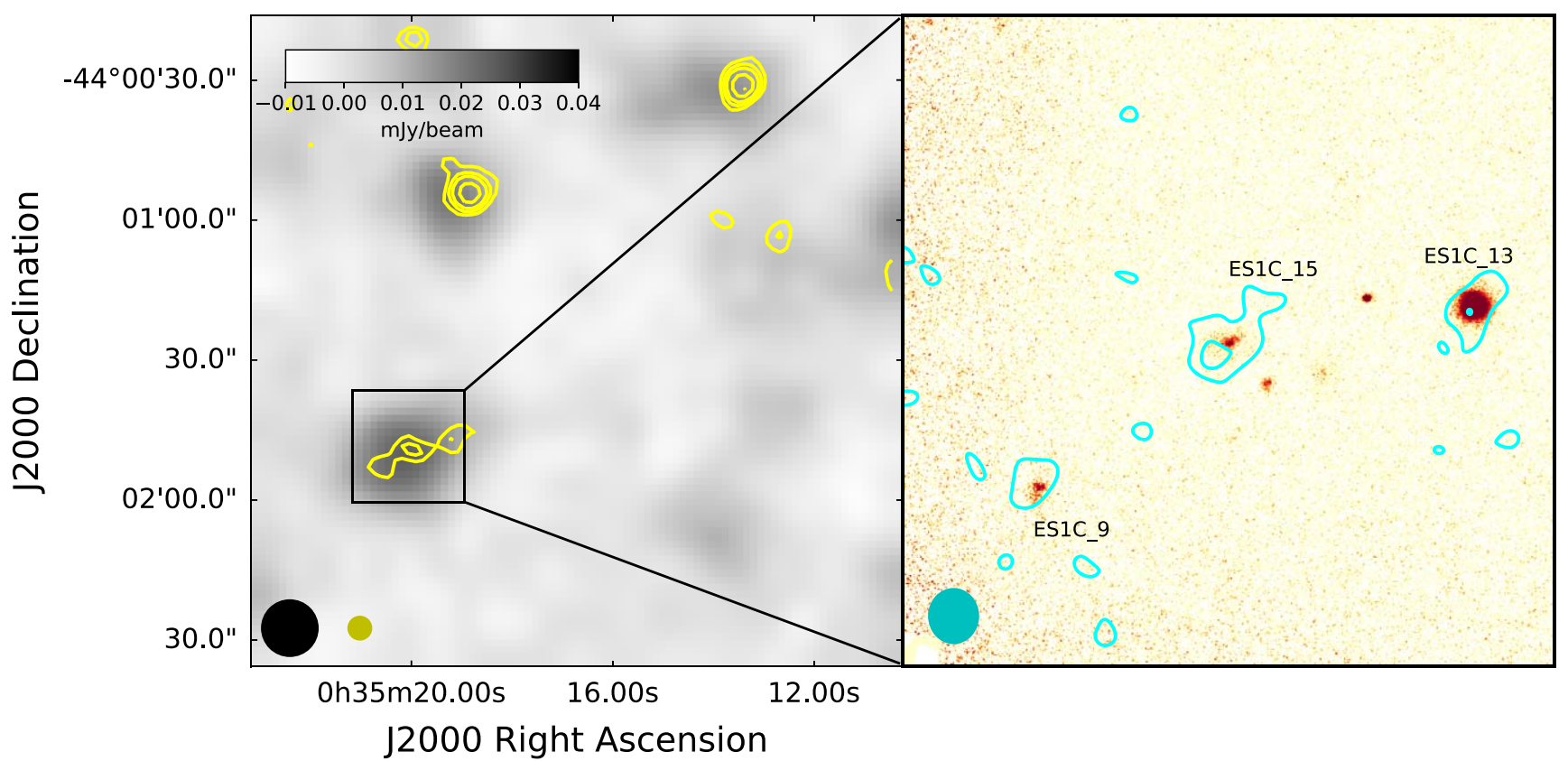

Figure 8. ES1C_H1. The left panel shows a grayscale image of the Herschel/SPIRE $250 \mu \mathrm{m}$ image of the ES1-C field with the overlaid contours of the SWIRE MIPS $24 \mu \mathrm{m}$ in yellow (levels $0.1,0.15,0.2,0.3$ and $0.4 \mathrm{MJy} \mathrm{sr}^{-1}$ ). The right panel shows a zoomed-in view of the region around ES1C_H1, showing the Gemini image overlaid ATCA $8.4 \mathrm{GHz}$ contours from the naturally weighted image in cyan at 3.6 and $7.2 \mu \mathrm{Jy}$ beam $^{-1}$. Beam sizes (FWHM) are indicated in the bottom left of each image.

Table 6

Surface Brightness Profile Demographics

\begin{tabular}{lcc}
\hline \hline Redshift Range & Exponential & de Vaucouleurs \\
\hline $0.5<z<1.0$ & $23 / 30$ & $7 / 9$ \\
$1.0<z<2.0$ & $4 / 6$ & $17 / 22$ \\
$2.0<z<4.0$ & $1 / 1$ & $16 / 17$ \\
\hline
\end{tabular}

Note. Redshift dependence of best-fitting surface brightness profile from our Tractor photometry. Sources best fit by exponential profiles are disk galaxies with high star formation rates, while sources best fit by de Vaucouleurs profiles tend to be bulge-dominated, quiescent galaxies.

whereas the star-forming galaxies are predominantly disks (Table 6).

Comparing to the CANDELS results of van der Wel et al., shown as the faint dots in Figure 5, we do see a larger fraction of compact star-forming galaxies, particularly at $z>2$. Only one of the $13 \quad z>2$ star-forming galaxies with masses $>10^{10} M_{\odot}$ in our study has a radius $>2 \mathrm{kpc}$, compared to 337 out of $774(43 \%)$ in CANDELS-COSMOS. This may be a selection effect due to lower surface brightness sensitivity in the Gemini data causing us to miss extended disks, though at high redshift the radii of the galaxies are close to the HST resolution limit of $\approx 0.7 \mathrm{kpc}$ (HWHM), suggesting that the limited resolution of HST may also play a role. Deeper Gemini observations would help us to better understand this issue.

\subsection{Galaxies Detected by Herschel/SPIRE}

The four Herschel sources (two in ES1-C and two in XMM-C2, see Figures 8-11) are comprised of multiple galaxies, some with similar photometric redshifts and some with very different ones. The Herschel images suffer from source confusion, so blending of sources that may or may not be physically associated is common. The details of the
Herschel detections are given in Table 7; see Section 3.6 for details on the deblending. The SEDs were fit as described in Section 3.8.

\subsubsection{ESIC_H1}

This source (Figure 8 ) has three $24 \mu \mathrm{m}$ sources apparently associated with it, which are in turn associated with galaxies ES1C_9, ES1C_13, and ES1C_15. ES1C_9 ( $\left.z_{\text {phot }}=3.0\right)$ appears to have an irregular morphology, with a compact core and more diffuse emission to its south (Figure 1, bottom left inset). It is brighter at $350 \mu \mathrm{m}$ than $250 \mu \mathrm{m}$ (Figure 6), and barely detected at $10 \mathrm{GHz}$, consistent with its relatively high redshift. ES1C_13 $\left(z_{\text {phot }}=0.3\right)$ is relatively bright at $24 \mu \mathrm{m}$ and detected at $10 \mathrm{GHz}$, but seems to not contribute to the Herschel source, consistent with its low redshift. ES1C_15 $\left(z_{\text {phot }}=2.3\right)$ is another disturbed looking galaxy, possibly in a group (though ES1C_12, the most nearby candidate companion, has a lower photometric redshift of 1.6). ES1C_15 dominates the $250 \mu \mathrm{m}$ flux and it is detected at $10 \mathrm{GHz}$ (Figure 1, bottom right, and Figure 8). The peaks of both the infrared and radio emission are offset to the southeast of the stellar light by about $0 . " 5$. In the HerMES catalog, one source is between ES1C_13 and ES1C_15 (4HERMES S250 SF J003519.2-440145) and one is between ES1C_9 and ES1C_15 (4HERMES S250 SF J003520.4-440151).

\subsection{2. $E S 1 C \_H 2$}

Several galaxies are visible within the contours of this source (Figure 1, top left, and Figure 9). Two are associated with a relatively strong $24 \mu \mathrm{m}$ source, ES1C_60 and ES1C_62, and also with weak radio sources, so we assume these are the dominant contributors to the Herschel flux. A third source, ES1C_55 (R.A. $(\mathrm{J} 2000)=00: 35: 18.73$, decl.(J2000) $=-44: 00: 57.3)$, is associated with a relatively strong radio source $\left(S_{8.4 \mathrm{GHz}}=175 \pm 5 \mu \mathrm{Jy}\right)$, but has no visible $24 \mu \mathrm{m}$ emission and is most likely a radio-loud 


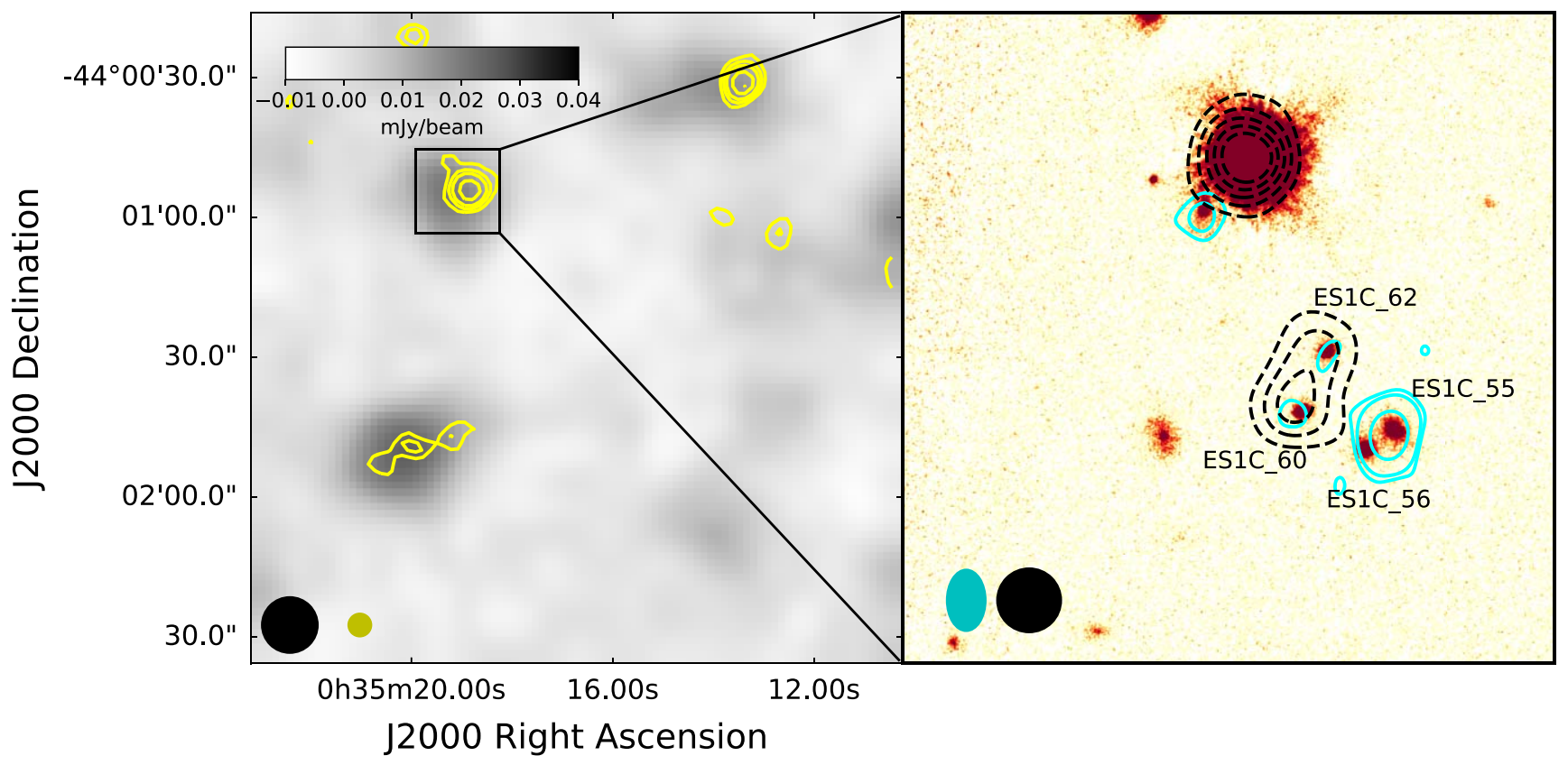

Figure 9. ES1C_H2. The left panel shows a grayscale image of the Herschel/SPIRE $250 \mu \mathrm{m}$ image of the ES1-C field with overlaid contours of the SWIRE MIPS $24 \mu \mathrm{m}$ in yellow (levels $0.1,0.15,0.2,0.3$, and $0.4 \mathrm{MJy} \mathrm{sr}^{-1}$ ). The right panel shows a zoomed-in view of the region around ES1C_H2, showing the Gemini image, overlaid with ATCA $8.4 \mathrm{GHz}$ contours from the uniform-weighted image in cyan at 6,12 , and $60 \mu \mathrm{Jy} /$ beam, and SWIRE $8 \mu \mathrm{m}$ emission (dashed) in black (contour levels $7.75,7.8,7.85,8.2$, and $8.4 \mathrm{MJy} \mathrm{sr}^{-1}$ ). Beam sizes (FWHM) are indicated in the bottom left of each image.

Table 7

Counterparts of AGN and Herschel Sources

\begin{tabular}{|c|c|c|c|c|c|c|c|c|c|}
\hline $\begin{array}{l}\text { Source } \\
\text { (1) }\end{array}$ & $\begin{array}{l}\text { R.A. } \\
\text { (J2000) } \\
(2)\end{array}$ & $\begin{array}{l}\text { Decl. } \\
(\mathrm{J} 2000) \\
(3)\end{array}$ & $\begin{array}{c}S_{5.8 \mu \mathrm{m}} \\
(\mu \mathrm{Jy}) \\
(4)\end{array}$ & $\begin{array}{c}S_{8.0 \mu \mathrm{m}} \\
(\mu \mathrm{Jy}) \\
(5)\end{array}$ & $\begin{array}{c}S_{24 \mu \mathrm{m}} \\
(\mu \mathrm{Jy}) \\
(6)\end{array}$ & $\begin{array}{l}S_{250 \mu \mathrm{m}} \\
(\mathrm{mJy}) \\
(7)\end{array}$ & $\begin{array}{l}S_{350 \mu \mathrm{m}} \\
(\mathrm{mJy}) \\
(8)\end{array}$ & $\begin{array}{c}S_{8.4 \mathrm{GHz}} \\
(\mu \mathrm{Jy}) \\
(9)\end{array}$ & $\begin{array}{l}q_{\mathrm{IR}} \\
(10)\end{array}$ \\
\hline \multicolumn{10}{|c|}{ ES1C_H1 (4HERMES S250 SF J003519.2-440145 and 4HERMES S250 SF J003520.4-440151) } \\
\hline ES1C_9 & 00:35:20.79 & $-44: 01: 55.7$ & $13 \pm 20$ & $4 \pm 20$ & $152 \pm 50$ & $5 \pm 4$ & $12 \pm 5$ & $9 \pm 4$ & 2.7 \\
\hline ES1C_13 & 00:35:20.11 & $-44: 01: 50.1$ & $22 \pm 20$ & $132 \pm 20$ & $177 \pm 50$ & $(-3 \pm 4)$ & $(-1 \pm 5)$ & $15 \pm 6$ & $\cdots$ \\
\hline ES1C_15 & 00:35:19.24 & $-44: 01: 48.6$ & $46 \pm 20$ & $46 \pm 20$ & $199 \pm 50$ & $30 \pm 4$ & $18 \pm 5$ & $15 \pm 5$ & 2.7 \\
\hline \multicolumn{10}{|c|}{ ES1C_H2 (4HERMES S250 SF J003519.2-440055) } \\
\hline ES1C_60 & $00: 35: 18.99$ & $-44: 00: 56.7$ & $113 \pm 20$ & $170 \pm 20$ & $500 \pm 50^{\mathrm{a}}$ & $27 \pm 4^{\mathrm{a}}$ & $18 \pm 5^{\mathrm{a}}$ & $10.0 \pm 2.4$ & $3.1^{\mathrm{a}}$ \\
\hline ES1C_62 & $00: 35: 18.92$ & $-44: 00: 54.8$ & $92 \pm 20$ & $126 \pm 20$ & $\ldots$ & $\cdots$ & $\cdots$ & $10.0 \pm 2.4$ & $\cdots$ \\
\hline \multicolumn{10}{|c|}{ XMM2_H1 (4HERMES S250 SF J022740.5-043322) } \\
\hline XMMC2_69 & $02: 27: 40.69$ & $-04: 33: 23.4$ & $120 \pm 20$ & $84 \pm 20$ & $151 \pm 50$ & $23 \pm 5$ & $17 \pm 8$ & $48 \pm 12^{\mathrm{b}}$ & 1.9 \\
\hline \multicolumn{10}{|c|}{ XMM2_H2 (4HERMES S250 SF J022742.4-043411) } \\
\hline XMMC2_30 & $02: 27: 43.52$ & $-04: 34: 10.9$ & $54 \pm 20$ & $40 \pm 20$ & $277 \pm 50$ & $19 \pm 5$ & $11 \pm 8$ & $<10^{\mathrm{b}}$ & 3.0 \\
\hline
\end{tabular}

Notes. Column (1): source name. Column (2): R.A. Column (3): Decl. Column (4): Spitzer $5.8 \mu \mathrm{m}$ flux from SWIRE. Column (5): Spitzer $8.0 \mu \mathrm{m}$ flux from SWIRE. Column (6): Spitzer $24 \mu \mathrm{m}$ flux from SWIRE. Column (7): Herschel $250 \mu \mathrm{m}$ flux from HERMES. Column (8): Herschel $350 \mu \mathrm{m}$ flux from HERMES. Column (9): $8.4 \mathrm{GHz}$ flux from our new radio observations. The data are from the ATCA, unless otherwise noted. Upper limits are given at the $3 \sigma$ level. Column (10): radioinfrared ratio.

${ }^{a}$ ES1C_60 and ES1C_62 have a separation of $\sim 2^{\prime \prime}$, and are thus too close to be deblended in the 24,250 , and $350 \mu \mathrm{m}$ bands. Thus, the infrared fluxes and $q_{\mathrm{IR}}$ correspond to the sum of the two objects.

${ }^{\mathrm{b}}$ Calculated based on the $8-12 \mathrm{GHz}$ VLA flux density and converted to $8.4 \mathrm{GHz}$ assuming a radio spectral index of $\alpha=-0.7$.

AGN. It has a close companion galaxy (ES1C_56). Finally, there is also a radio source very close $\left(\approx 2^{\prime \prime} \mathrm{SE}\right)$ to the guide star (unfortunately too close to be able to obtain reliable optical/ infrared photometry).

Both ES1C_60 and ES1C_62, which appear as a close pair on the sky, have SEDs consistent with a dominant AGN component. ES1C_62 has a point-source contribution approximately equal in flux to that of the extended host galaxy, so it is most likely a Type-1 (unobscured) AGN. All three AGN (ES1C_55, ES1C_60 and ES1C_62) have similar photometric redshifts $(1.37,1.45$, and 1.44 for ES1C_55, ES1C_60, and ES1C_62, respectively, and ES1C_56, the non-AGN companion to ES1C_55, is at a redshift of 1.49.) Thus, this system is a candidate triple-AGN (which needs to be confirmed via spectroscopy). Only a handful of these are known (e.g., Liu et al. 2011; Schawinski et al. 2011; Deane et al. 2014). Unfortunately, its proximity to one of the guide stars will make spectroscopic confirmation of this system challenging. The guide star is $6^{\prime \prime}$ from the nearest AGN (ES1C_62); if scattered light can be excluded then a conventional longslit spectrum 
Table 8

Derived Properties of Herschel Sources

\begin{tabular}{|c|c|c|c|c|c|c|c|c|}
\hline $\begin{array}{l}\text { Source } \\
\text { (1) }\end{array}$ & $\begin{array}{l}z_{\text {phot }} \\
(2)\end{array}$ & $\begin{array}{c}L_{\mathrm{IR}} \\
\left(10^{12} L_{\odot}\right) \\
(3)\end{array}$ & $\begin{array}{c}\mathrm{SFR}(\mathrm{IR}) \\
\left(M_{\odot} \mathrm{yr}^{-1}\right) \\
(4)\end{array}$ & $\begin{array}{c}L_{1.4 \mathrm{GHz}} \\
\left(\mathrm{W} \mathrm{Hz}^{-1}\right) \\
(5)\end{array}$ & $\begin{array}{c}\text { SFR (radio) } \\
\left(M_{\odot} \mathrm{yr}^{-1}\right) \\
(6)\end{array}$ & $\begin{array}{c}r_{1 / 2} \\
(\mathrm{kpc}) \\
(7)\end{array}$ & $\begin{array}{c}M_{*} \\
\left(M_{\odot}\right) \\
(8)\end{array}$ & $\begin{array}{c}\text { Model } \\
\text { (9) }\end{array}$ \\
\hline \multicolumn{9}{|l|}{$\overline{\text { ES1C_H1 }}$} \\
\hline ES1C_15 & 2.33 & 5.0 & 860 & $2.4 \times 10^{24}$ & 1300 & 3.8 & $3.1 \times 10^{11}$ & Exp \\
\hline ES1C_H2 & & 2.6 & $180^{\mathrm{a}}$ & & & & & \\
\hline ES1C_62 & 1.44 & $\ldots$ & $\cdots$ & $5.2 \times 10^{23}$ & 290 & 0.4 & $1.2 \times 10^{11}$ & Exp \\
\hline \multicolumn{9}{|l|}{ XMM2_H1 } \\
\hline XMMC2_69 & 1.61 & 1.1 & 190 & $2.9 \times 10^{24}$ & 1600 & 10.5 & $7.9 \times 10^{10}$ & Dev \\
\hline \multicolumn{9}{|l|}{ XMM2_H2 } \\
\hline XMMC2_30 & 2.01 & 1.8 & 310 & $<1.0 \times 10^{24}$ & $<560$ & 1.9 & $1.2 \times 10^{11}$ & Exp \\
\hline
\end{tabular}

Note.

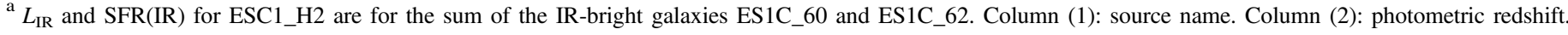

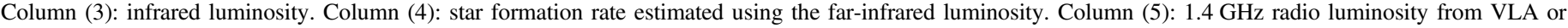

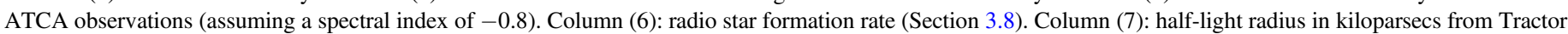

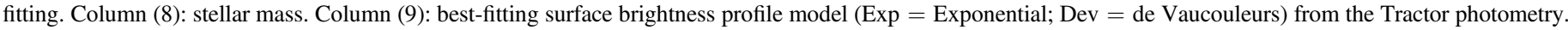

from a large ground-based telescope might be practical, but this system is probably better investigated using a space-based platform, or a spectrograph fed by AO. Even so, care will need to be taken to ensure stray light from the star does not affect the observation.

Fitting the Composite-2 SED of Kirkpatrick et al. (2015; the closest template presented in that paper, though still failing to capture some of the mid-infrared flux) suggests that the infrared emission from star formation (60\% of the total) corresponds to a star formation rate summed over the whole system of about $180 M_{\odot} \mathrm{yr}^{-1}$ (Table 8). As star formation dominates the farinfrared flux, it is likely that this is a good estimate despite the relatively poor fit in the mid-infrared. The star formation rate estimate based on the radio emission is significantly higher, $\approx 600 M_{\odot} \mathrm{yr}^{-1}$ (excluding the emission from the radio galaxy, ES1C_55). However, it is likely that at least some of the radio emission is from the AGN even in the "radio-quiet" systems. In the HerMES catalog, this source is identified with 4HERMES S250 SF J003519.2-440055, from a $24 \mu \mathrm{m}$ prior that appears to be dominated by the blend of ES1C_60 and ES1C_62.

We used the Tractor to decompose the light from the Type-1 AGN ES1C_62 in the Gemini $K$-band data (Figure 12), the only AGN to show a point-source nucleus. Two components were fit, a point-source nucleus representing the AGN, and an extended host galaxy. The best fit indicates the AGN and the host galaxy each contribute $\approx 18 \pm 4 \mu \mathrm{Jy}$ to the total flux of the object, with the host being best fit by a fairly compact disk galaxy $(0$ " $05 \pm 0.02$ half-light radius). The point-source flux corresponds to $M_{i} \approx-23.3$ at $z_{\text {phot }}=1.44$. This object, and its companion Type-2 AGN host ES1C_60 (which has a very similar luminosity and a half-light radius of $\approx 0$ ". 1 ), can be compared to the $z \approx 1.8$ faint quasars studied with the NICMOS instrument on HST by Ridgway et al. (2001). In general, the host galaxies in the Ridgway et al. sample are fainter $\left(M_{i} \sim-21\right.$ to 22 compared to $\left.M_{i} \approx-23.3\right)$, but have larger scale sizes ( $\sim 2 \mathrm{kpc}$ compared to $\approx 0.4-0.8 \mathrm{kpc}$ ). Whether these differences are due to small number statistics, different host properties of infrared versus optically selected AGNs, or due to the difficulty of removing the larger HST PSF, remains to be established.

\subsection{3. $X M M C 2-H 1$}

This source is identified with a pair of interacting galaxies (XMMC2_69 and XMMC2_67; upper left inset in Figure 2) with a mean photometric redshift of $z_{\text {phot }} 1.5$ (1.42 and 1.60 for XMMC2_69 and XMMC2_67, respectively). A faint detection in the VLA data (Figure 10) indicates that the bulk of the $\approx 190 M_{\odot} \mathrm{yr}^{-1}$ of star formation is in XMMC2_69. In the HerMES catalog this source is identified with 4HERMES S250 SF J022740.5-043322.

\subsubsection{XMMC2_H2}

The near-infrared emission from this source at $z_{\text {phot }}=2.01$ seems significantly offset from the 8 and $24 \mu \mathrm{m}$ peak (Figure 11), suggesting a highly obscured star-forming companion that is not seen, even in the IRAC $4.5 \mu \mathrm{m}$ data from SERVS. The estimated star formation rate of $310 M_{\odot} \mathrm{yr}^{-1}$ based on the Herschel far-infrared flux is consistent with the limit from the lack of a radio detection of $<560 M_{\odot} \mathrm{yr}^{-1}$. In the HerMES catalog this source is identified with 4HERMES S250 SF J022742.4-043411.

\subsection{Other Individual Objects}

We briefly mention some other interesting galaxies highlighted in Figures 1-3, and listed in Table 9. ES1C_46 is a disturbed disk galaxy with $z_{\text {phot }}=0.9$; it is weakly detected at $24 \mu \mathrm{m}$. The CDFS-C field has the best image quality of our fields, but unfortunately lacks Herschel detections. Nevertheless, we identify several high-redshift galaxies (Figure 2). CDFSC_60 is a compact, $z_{\text {phot }}=3.2$ galaxy with a half-light radius of 0 !'032 $(0.24 \mathrm{kpc})$, and CDFSC_11 and CDFSC_12 are an apparent pair of galaxies at $z_{\text {phot }}=2.9$. 


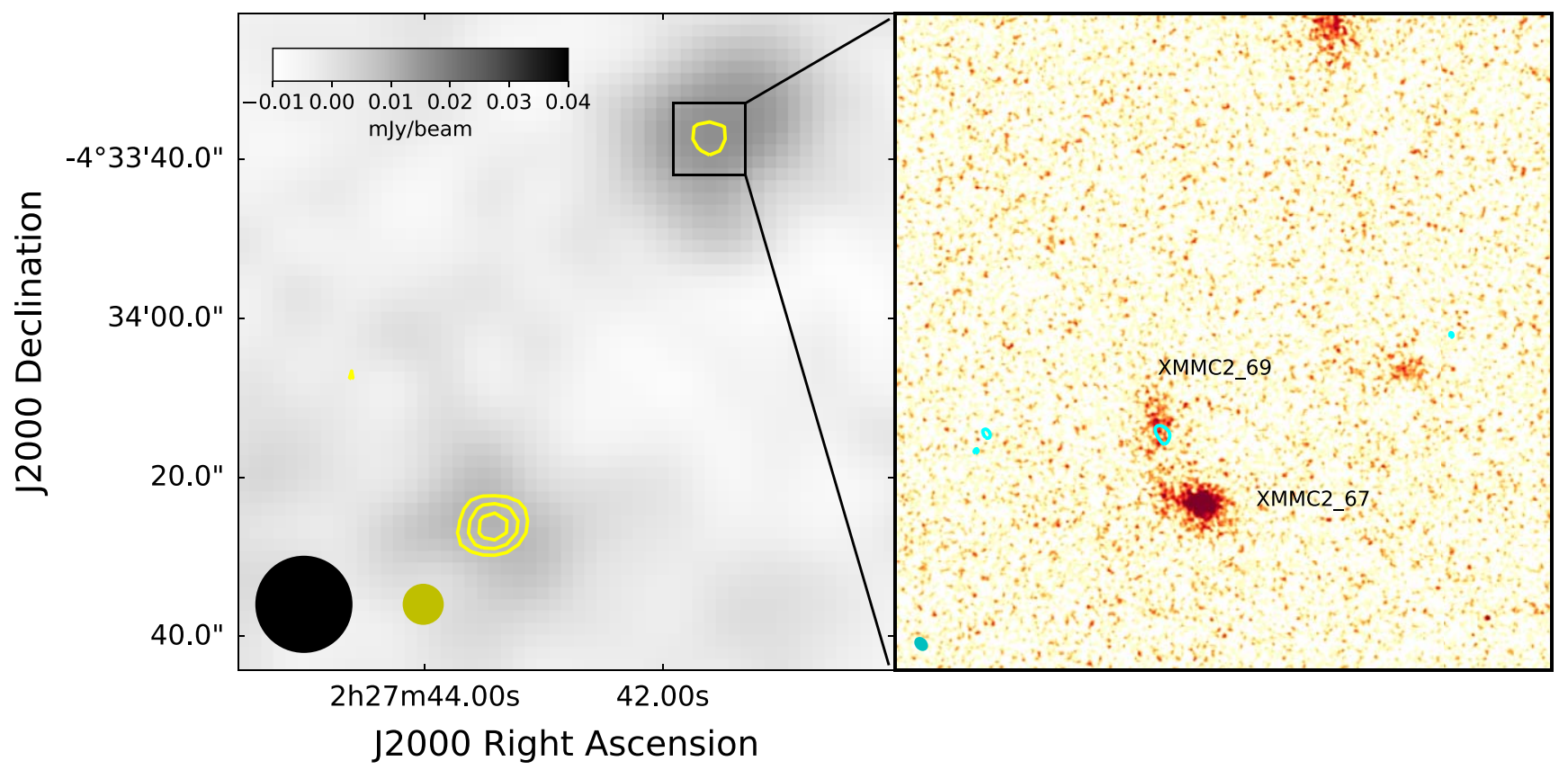

Figure 10. XMMC2_H1. The left panel shows a grayscale image of the Herschel/SPIRE $250 \mu \mathrm{m}$ image of the XMMC-2 field with overlaid contours of the SWIRE MIPS $24 \mu \mathrm{m}$ in yellow (levels $0.1,0.15$ and $0.2 \mathrm{MJy} \mathrm{s}^{-1}$ ) above background). The right panel shows a zoomed-in view of the region around XMMC2_H1, showing the Gemini image overlaid with the $10 \mu \mathrm{Jy}$ beam $^{-1}$ contour from the naturally weighted VLA $10 \mathrm{GHz}$ image in cyan. Beam sizes (FWHM) are indicated in the bottom left of each image.

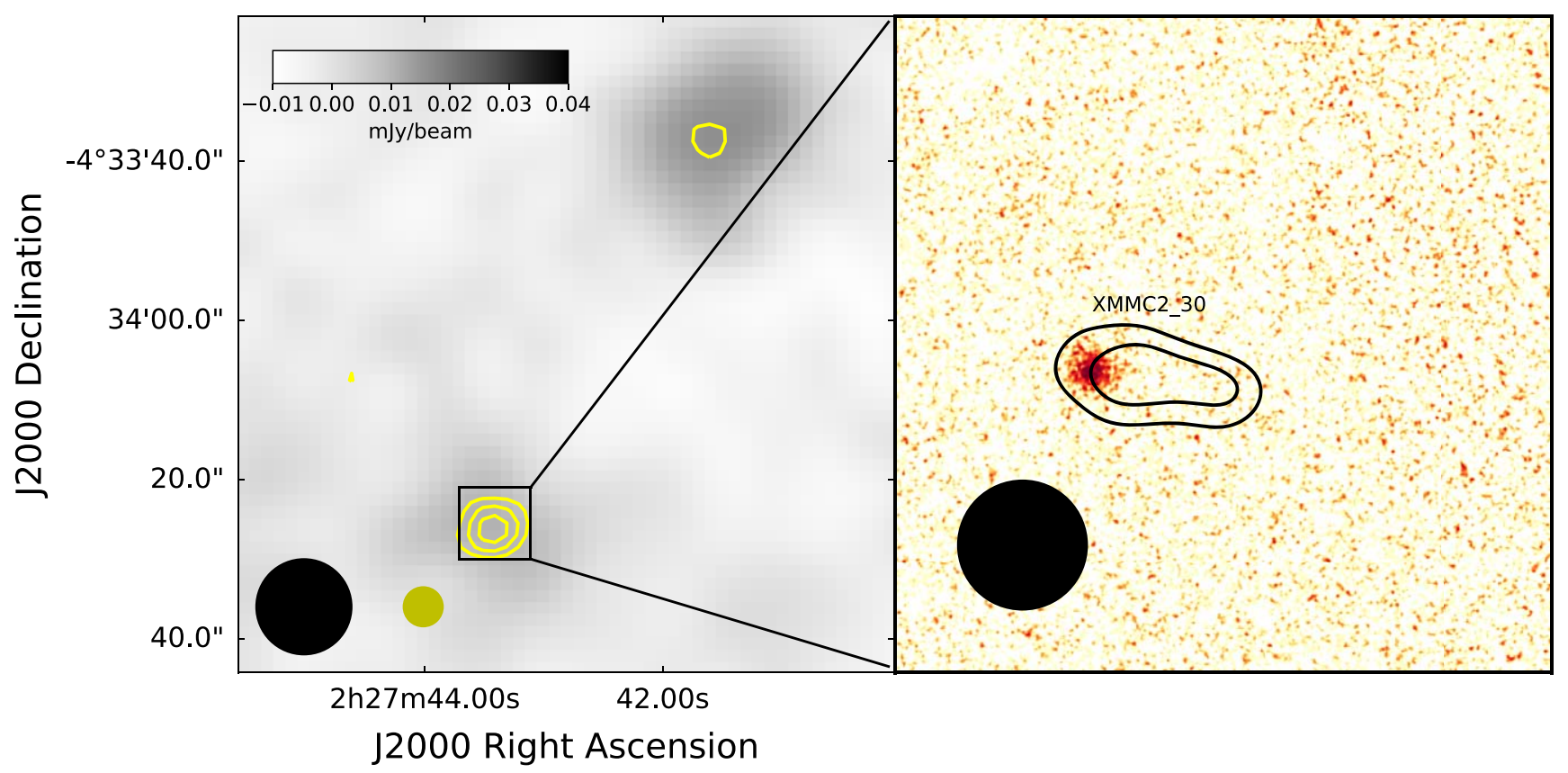

Figure 11. XMMC2_H2. The left panel shows a grayscale image of the Herschel/SPIRE $250 \mu \mathrm{m}$ image of the XMM-C2 field with overlaid contours of the SWIRE MIPS $24 \mu \mathrm{m}$ in yellow (at levels of 0.1, 0.15, 0.2, 0.3, and $0.4 \mathrm{MJy} \mathrm{sr}^{-1}$ ). The right panel shows a zoomed-in view of the region around XMMC2_H2, showing the Gemini image overlaid with contours from the SWIRE $8 \mu \mathrm{m}$ image in black (contour levels 7.75 and $7.8 \mathrm{MJy} \mathrm{sr}^{-1}$ ). Beam sizes (FWHM) are indicated in the bottom left corner of each image.

\section{Discussion}

We have presented some of the highest angular resolution images of $z \gtrsim 1$ galaxies obtained in the near-infrared. Our results on the evolution of galaxy sizes are broadly consistent with larger studies made with the HST (van der Wel et al. 2014; Shibuya et al. 2015; Allen et al. 2017), though we see some differences, particularly in the size distribution of moderately massive $>10^{10} M_{\odot}$ star-forming galaxies at $z>2$, where we see a higher fraction of compact (radius $<2 \mathrm{kpc}$ ) objects $(\approx 90 \%$ of the population in this study compared to $\approx 60 \%$ with $H S T)$ that will need further Gemini data, or observations with the JWST to resolve.

The launch of JWST will enable studies of large numbers of the field galaxy population at similar angular resolution and very high sensitivity, but the difficulty of performing large surveys with JWST will leave a niche for ground-based MCAO 


\section{Original Image}

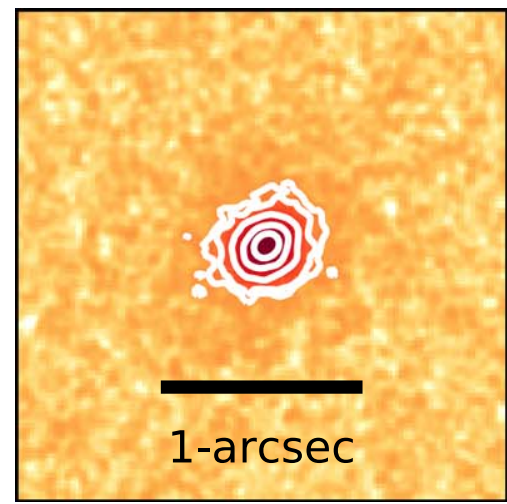

Model Galaxy+Nucleus

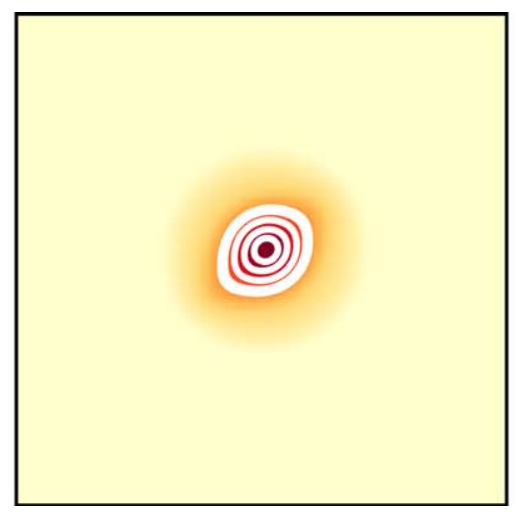

Residual

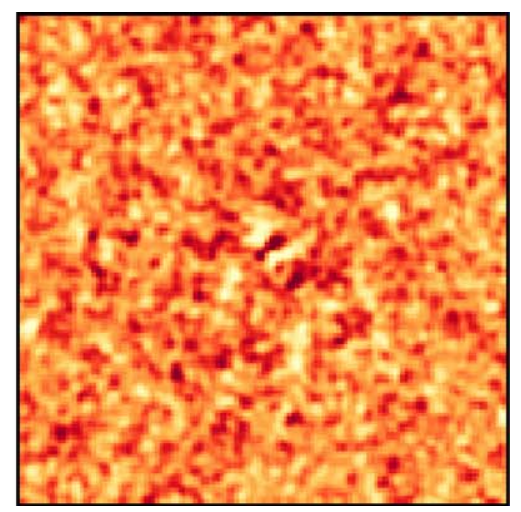

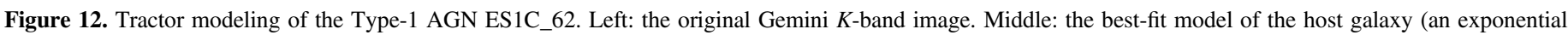

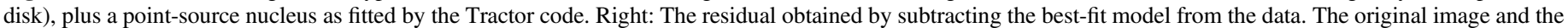
model are shown with a logarithmic stretch and the residual image is shown with a linear stretch. Contours are at 3, 4, 8, 16, and 32 times the rms noise.

Table 9

Other Objects of Interest

\begin{tabular}{lcccc}
\hline \hline Source & $z_{\text {phot }}$ & $\begin{array}{c}M_{*} \\
\left(M_{\odot}\right)\end{array}$ & $\begin{array}{c}r_{1 / 2} \\
(\mathrm{kpc})\end{array}$ & Model \\
$(1)$ & $(2)$ & $(3)$ & $(4)$ & $(5)$ \\
\hline ES1C_46 & 0.9 & $2.5 \times 10^{10}$ & 3.8 & Exp \\
CDFSC_60 & 3.2 & $2.0 \times 10^{10}$ & 0.24 & Dev \\
CDFSC_11 & 2.9 & $1.3 \times 10^{10}$ & 1.1 & Exp \\
CDFSC_12 & 2.9 & $4.0 \times 10^{10}$ & 0.5 & Exp \\
\hline
\end{tabular}

Note. Column (1): source name. Column (2): photometric redshift. Column (3): stellar mass. Column (4): half-light radius in kiloparsecs from the Tractor fitting. Column (5): best-fitting surface brightness profile model $(\operatorname{Exp}=\mathrm{Ex}-$ ponential; Dev $=$ de Vaucouleurs) from the Tractor photometry.

to make targeted observations of large numbers of rare objects. We have therefore emphasized the study of the hosts of ULIRGs in our fields. By combining the Gemini data with infrared data from Spitzer and Herschel, together with arcsecond or better resolution radio data, we show that most of the Herschel sources appear to arise from multiple systems, including one candidate triple-AGN that would not have been otherwise identified as a multiple system. ${ }^{22}$ The high resolution of GeMS at wavelengths in the rest-frame optical is thus an essential need for accurately classifying these systems. For example, Capelo et al. (2017) use simulations to estimate that $\sim 20 \%$ of $\mathrm{AGN}$ are dual, but that high resolution $(\lesssim 10 \mathrm{kpc})$ data are needed to identify them. Observations such as these can therefore significantly improve the constraints on galaxy and supermassive black hole merger rates.

Figure 5 compares the counterparts of the Herschel sources to field galaxies at similar redshifts. As expected, they tend to be the more massive galaxies. The corollary of this is that a large fraction, $\gtrsim 50 \%$ of the $\gtrsim 10^{11} M_{\odot}$ galaxies at $z>1$ in our sample, show strong AGN or starburst activity, which is also seen in other samples of massive high- $z$ galaxies (Spitler et al. 2014; Martis et al. 2016; Schreiber et al. 2017). Also, as expected, the Herschel galaxies fall on the red side of the starforming population, and the radio galaxy (ES1C_55) plots among the quiescent objects. The AGN hosts (ES1C_55,60 and

\footnotetext{
22 Although the triple-AGN system is resolved in ground-based imaging, it is cataloged as a single detection in the VIDEO near-infrared catalog.
}

62) also tend to have more compact scale sizes than the starforming galaxies, a trend that has also been noted for highredshift AGNs selected from WISE (Farrah et al. 2017).

Previous studies of Herschel and other high-redshift, submillimeter-selected galaxies found broadly similar results. Kartaltepe et al. (2012) examined the morphologies of 52 $0.5<z<2$ ULIRGs selected from the GOODS-S field at 100 and $160 \mu \mathrm{m}$ (using PACS photometry), finding that the merger fraction in HST F160W images was higher in the ULIRGs $(50 \%-73 \%)$ compared to a matched sample of field galaxies on the star-forming "main sequence" of star formation rate versus stellar mass (24\%). With only a few objects it is difficult to estimate a merger rate, but of the four Herschel sources we have examined, we have one close pair (XMM-C2_69), one candidate triple-AGN (ES1C_60, 62, and 55), and two objects at different redshifts associated with the same Herschel source with disturbed morphologies that might be late stage mergers (ES1C_9 and ES1C_15). Thus, we are certainly consistent with the results of Kartaltepe et al. (2012). In contrast, Targett et al. (2013) found few mergers in their HST/WFC3 study of $1<z<3$ galaxies selected in the submillimeter, but did find, as we do, that the $z>2$ star-forming ULIRG population is dominated by large-disk galaxies (in contrast to the AGNs in this sample, which have a mix of profiles and smaller half-light radii).

The Herschel systems studied in this paper can also be compared to the high-resolution studies of $z \sim 4$ star-forming galaxies by Wiklind et al. (2014), Hodge et al. (2016), and Rujopakarn et al. (2016) with HST and radio/submillimeter imaging with the VLA and ALMA, which reveal similar trends, namely disturbed host galaxy morphologies and starforming regions offset from the centroid of the stellar light (XMMC2_30 is a very good example). The fact that these morphologies persist into the $K$ band (rest-frame optical) in the sample studied in this paper suggest that the appearance of these objects is not dominated by clumpy extinction, but instead reflects the actual distribution of stellar light. The Herschel/SPIRE selected objects in this paper thus provide a bridge between objects discovered in deep Herschel/PACS observations at shorter wavelengths and mostly lower redshifts, and the more extreme objects (both in terms of luminosity and redshift) discovered in the submillimeter surveys.

A planned upgrade for GeMS will allow it to be used with guide stars as faint as $R \approx 17$. When enacted, this will open up 
a much larger area of the extragalactic sky to subarcsecondresolution near-infrared observations. Although ground-based imaging will not be able to compete with space-based observations from JWST in terms of depth, the ability to survey a large number of widely separated targets much more efficiently than JWST, and with a much less oversubscribed facility, will make GSAOI a useful tool for following up large numbers of rare objects. These might include luminous submillimeter galaxies and the hosts of luminous AGN/quasars. With a larger sample, we can test whether the trends suggested in this pilot study are present in the population as a whole.

We thank J.C. Mauduit for assistance with the CTIO observations in this paper. The National Radio Astronomy Observatory is a facility of the National Science Foundation operated under cooperative agreement by Associated Universities, Inc. This work made extensive use of TOPCAT (Taylor 2005) for catalog matching and analysis, and the Virtual Observatory SAMP protocol for communication between applications. This research made use of Montage. It is funded by the National Science Foundation under grant No. ACI1440620, and was previously funded by the National Aeronautics and Space Administration's Earth Science Technology Office, Computation Technologies Project, under Cooperative Agreement Number NCC5-626 between NASA and the California Institute of Technology. This research made use of APLpy, an open-source plotting package for Python (Robitaille \& Bressert 2012). J.A. gratefully acknowledges support from the Science and Technology Foundation (FCT, Portugal) through the research grant UID/FIS/04434/2013.

\section{ORCID iDs}

M. Lacy (1) https://orcid.org/0000-0002-3032-1783

P. Jagannathan (1) https://orcid.org/0000-0002-5825-9635

J. Afonso (i) https://orcid.org/0000-0002-9149-2973

D. Farrah (1) https://orcid.org/0000-0003-1748-2010

A. O. Petric (1) https://orcid.org/0000-0003-4030-3455

A. Sajina (1) https://orcid.org/0000-0002-1917-1200

J. A. Surace 1 il https://orcid.org/0000-0001-7291-0087

M. Vaccari (i) https://orcid.org/0000-0002-6748-0577

\section{References}

Allen, R. J., Kacprzak, G. G., Glazebrook, K., et al. 2017, ApJL, 834, L11 Bell, E. F. 2003, ApJ, 586, 794

Berriman, G. B., Good, J. C., Curkendall, D., et al. 2003, in ASP Conf. Ser. 295, adass XII, ed. H. E. Payne, R. I. Jedrzejewski, \& R. N. Hook (San Francisco, CA: ASP), 343

Bertin, E. 2006, in ASP Conf. Ser. 351, adass XV, ed. C. Gabriel et al. (San Francisco, CA: ASP), 112

Bertin, E., \& Arnouts, S. 1996, A\&AS, 117, 393

Bluck, A. F. L., Conselice, C. J., Buitrago, F., et al. 2012, ApJ, 747, 34 Bolzonella, M., Miralles, J.-M., \& Pello, R. 2000, A\&A, 363, 476 Brammer, G. B., van Dokkum, P. G., \& Coppi, P. 2008, ApJ, 686, 1503 Buitrago, F., Trujillo, I., Conselice, C. J., et al. 2008, ApJL, 687, L61 Calzetti, D., Kinney, A. L., \& Storchi-Bergmann, T. 1994, ApJ, 429, 582 Capelo, P. R., Dotti, M., Volonteri, M., et al. 2017, MNRAS, 469, 4437 Cappellari, M. 2016, ARA\&A, 54, 597

da Cunha, E., Charlot, S., \& Elbaz, D. 2008, MNRAS, 388, 1595

Daddi, E., Renzini, A., Pirzkal, N., et al. 2005, ApJ, 626, 680

Dark Energy Survey Collaboration, Abbott, T., Abdalla, F. B., et al. 2016, MNRAS, 460, 1270

Deane, R. P., Paragi, Z., Jarvis, M. J., et al. 2014, Natur, 511, 57

Dekel, A., \& Burkert, A. 2014, MNRAS, 438, 1870
Delhaize, J., Smolčić, V., Delvecchio, I., et al. 2017, A\&A, 602, 4 Farrah, D., Petty, S., Connolly, B., et al. 2017, ApJ, 844, 106 Furlong, M., Bower, R. G., Crain, R. A., et al. 2017, MNRAS, 465, 722 Gwyn, S. D. J. 2012, AJ, 143, 38

Glassman, T. M., Larkin, J. E., \& Lafrenière, D. 2002, ApJ, 581, 865 Helou, G., Soifer, B. T., \& Rowan-Robinson, M. 1985, ApJL, 298, L7 Hodge, J. A., Swinbank, A. M., Simpson, J. M., et al. 2016, ApJ, 822, 103 Hogg, D. W., \& Lang, D. 2013, PASP, 125, 719

Hurley, P. D., Oliver, S., Betancourt, M., et al. 2017, MNRAS, 464, 885 Ivison, R. J., Magnelli, B., Ibar, E., et al. 2010, A\&A, 518, L31 Jarvis, M. J., Bonfield, D. G., Bruce, V. A., et al. 2013, MNRAS, 428, 1281 Kartaltepe, J. S., Dickinson, M., Alexander, D. M., et al. 2012, ApJ, 757, 23 Kirkpatrick, A., Pope, A., Sajina, A., et al. 2015, ApJ, 814, 9

Kriek, M., \& Conroy, C. 2013, ApJL, 775, L16

Lacy, M., Storrie-Lombardi, L. J., Sajina, A., et al. 2004, ApJS, 154, 166

Lang, D., Hogg, D. W., \& Schlegel, D. J. 2016, AJ, 151, 36

Liu, X., Shen, Y., \& Strauss, M. A. 2011, ApJL, 736, L7

Lonsdale, C., Smith, H. E., Rowan-Robinson, M., et al. 2003, PASP, 115, 897

Magnelli, B., Ivisonm, R. J., Lutz, D., et al. 2015, A\&A, 573, 45

Mao, M. Y., Huynh, M. T., Norris, R. P., et al. 2011, ApJ, 731, 79

Maraston, C. 2005, MNRAS, 362, 799

Maraston, C., Daddi, E., Renzini, A., et al. 2006, ApJ, 652, 85

Maraston, C., Pforr, J., Renzini, A., et al. 2010, MNRAS, 407, 830

Martis, N. S., Marchesini, D., Brammer, G. B., et al. 2016, ApJL, 827, L25

Mauduit, J.-C., Lacy, M., Farrah, D., et al. 2012, PASP, 124, 1135

McMullin, J. P., Waters, B., Schiebel, D., Young, W., \& Golap, K. 2007, in ASP Conf. Ser. 376, adass XVI, ed. R. A. Shaw, F. Hill, \& D. J. Bell (San Francisco, CA: ASP), 127

Melbourne, J., Peng, C. Y., Soifer, B. T., et al. 2011, AJ, 141, 141 Murphy, E. J., Momjian, E., Condon, J. J., et al. 2017, ApJ, 839, 35 Nayyeri, H., Hemmati, S., Mobasher, B., et al. 2017, ApJS, 228, 7 Neichel, B., Rigaut, F., Vidal, F., et al. 2014, MNRAS, 440, 1002 Newman, A. B., Ellis, R. S., Bundy, K., \& Treu, T. 2012, ApJ, 746, 162 Nyland, K., Lacy, M., Sajina, A., et al. 2017, ApJS, 230, 9

Oliver, S. J., Bock, J., Altieri, B., et al. 2012, MNRAS, 424, 1614

Pandya, V., Brennan, R., Somerville, R. S., et al. 2017, MNRAS, 472, 2054

Patel, S. G., Hong, Y. X., Quadri, R. F., Holden, B. P., \& Williams, R. J. 2017, ApJ, 839, 127

Perna, M., Brusa, M., Salvato, M., et al. 2015, A\&A, 583, A72 Petty, S. M., Neill, J. D., Jarrett, T. H., et al. 2013, AJ, 146, 77 Pforr, J., Maraston, C., \& Tonini, C. 2012, MNRAS, 422, 3285

Pforr, J., Vaccari, M., Lacy, M., et al. 2018, MNRAS, submitted

Pilbratt, G. L., Riedinger, J. R., Passvogel, T., et al. 2010, A\&A, 518, L1

Ridgway, S. E., Heckman, T. M., Calzetti, D., \& Lehnert, M. 2001, ApJ, 550, 122

Rigaut, F., Neichel, B., Boccas, M., et al. 2014, MNRAS, 437, 2361

Robitaille, T., \& Bressert, E. 2012, APLpy, Astrophysics Source Code Library, ascl: 1208.017

Rodighiero, G., Renzini, A., Daddi, E., et al. 2014, MNRAS, 443, 19

Rujopakarn, W., Dunlop, J. S., Rieke, G. H., et al. 2016, ApJ, 833, 12 Rujopakarn, W., Nyland, K., Rieke, G. H., et al. 2018, ApJ, 854, 4

Sault, R. J., Teuben, P. J., \& Wright, M. C. H. 1995, in ASP Conf. Ser. 77, adass

IV, ed. R. A. Shaw, H. E. Payne, \& J. J. E. Hayes (San Francisco, CA) 433 Schawinski, K., Urry, M., Treister, E., et al. 2011, ApJL, 743, L37 Schirmer, M., Carrasco, E. R., Pessev, P., et al. 2015, ApJS, 217, 33 Schreiber, C., Pannella, M., Leiton, R., et al. 2017, A\&A, 599, A134 Shibuya, T., Ouchi, M., \& Harikane, Y. 2015, ApJS, 219, 15

Speagle, J. S., Steinhardt, C. L., Capak, P. L., \& Silverman, J. D. 2014, ApJS, 214,15

Spitler, L. R., Straatman, C. M. S., Labbé, I., et al. 2014, ApJL, 787, L36 Sweet, S. M., Sharp, R., Glazebrook, K., et al. 2017, MNRAS, 464, 2910 Tallon, M., \& Foy, R. 1990, A\&A, 235, 549

Targett, T. A., Dunlop, J. S., Cirasuolo, M., et al. 2013, MNRAS, 432, 2012 Taylor, M. B. 2005, in ASP Conf. Ser. 347, Astronomical Data Analysis Software and Systems XIV, ed. P. Shopbell, M. Britton, \& R. Ebert (San Francisco, CA: ASP), 29

Turri, P., McConnachie, A. W., Stetson, P. B., et al. 2017, AJ, 153, 199 van der Wel, A., Bell, E. F., Häussler, B., et al. 2012, ApJS, 203, 24 van der Wel, A., Franx, M., van Dokkum, P. G., et al. 2014, ApJ, 788, 28 Wellons, S., Torrey, P., Ma, C.-P., et al. 2015, MNRAS, 449, 361 Whitaker, K. E., Labbé, I., van Dokkum, P. G., et al. 2011, ApJ, 735, 86 Wiklind, T., Conselice, C. J., Dahlen, T., et al. 2014, ApJ, 785, 111 Wuyts, S., Cox, T. J., Hayward, C. C., et al. 2010, ApJ, 722, 1666 